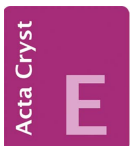

CRYSTALLOGRAPHIC COMMUNICATIONS

ISSN 2056-9890

\section{Channels with ordered water and bipyridine molecules in the porous coordination polymer $\left\{\left[\mathrm{Cu}\left(\mathrm{SiF}_{6}\right)\left(\mathrm{C}_{10} \mathrm{H}_{8} \mathrm{~N}_{2}\right)_{2}\right] \cdot 2 \mathrm{C}_{10} \mathrm{~N}_{2} \mathrm{H}_{8} \cdot 5 \mathrm{H}_{2} \mathrm{O}\right\}_{n}$}

Edited by A. Van der Lee, Université de Montpellier II, France

Keywords: Porous coordination polymer; adsorption; hydrogen bonding; $\pi-\pi$ stacking; copper(II); 4,4'-bipyridine; crystal structure.

CCDC reference: 1510381

Supporting information: this article has supporting information at journals.iucr.org/e

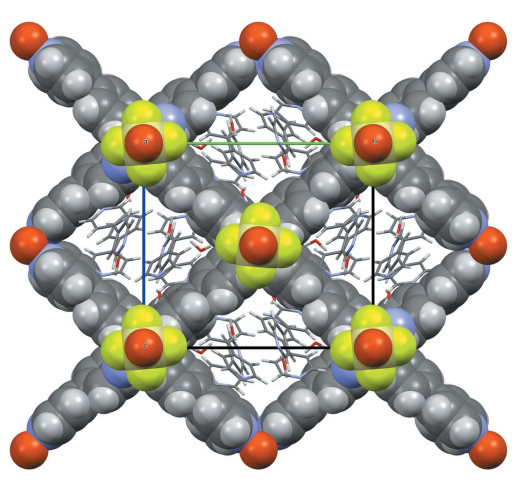

OPEN $\odot$ ACCESS

\author{
Emmanuel Aubert, ${ }^{\mathrm{a} *}$ Abdelatif Doudouh, ${ }^{\mathrm{a}}$ Paola Peluso $^{\mathrm{b}}$ and Victor Mamane ${ }^{\mathrm{c}}$
}

${ }^{a}$ Cristallographie, Résonance Magnétique et Modélisations (CRM2), UMR CNRS, 7036, Université de Lorraine, BP 70239 , Bd des Aiguillettes, 54506, Vandoeuvre-les-Nancy, France, 'b Istituto di Chimica Biomolecolare ICB CNR - Sede Secondaria di Sassari, Traversa La Crucca 3, Regione Baldinca, I-07100 Li Punti - Sassari, Italy, and ' Institut de Chimie de Strasbourg, UMR 7177, Equipe LASYROC, 1 rue Blaise Pascal, BP 296 R8, 67008 Strasbourg Cedex, France.

*Correspondence e-mail: emmanuel.aubert@univ-lorraine.fr

The coordination polymer $\left\{\left[\mathrm{Cu}\left(\mathrm{SiF}_{6}\right)\left(\mathrm{C}_{10} \mathrm{H}_{8} \mathrm{~N}_{2}\right)_{2}\right] \cdot 2 \mathrm{C}_{10} \mathrm{H}_{8} \mathrm{~N}_{2} \cdot 5 \mathrm{H}_{2} \mathrm{O}\right\}_{n}$, systematic name: poly[[bis $\left(\mu_{2}-4,4^{\prime}\right.$-bipyridine $)\left(\mu_{2}\right.$-hexafluoridosilicato)copper(II)] $4,4^{\prime}$-bipyridine disolvate pentahydrate], contains pores which are filled with water and 4,4'-bipyridine molecules. As a result of the presence of these ordered species, the framework changes its symmetry from $P 4 / \mathrm{mmm}$ to $P 2_{1} / c$. The $4,4^{\prime}$-bipyridine guest molecules form chains inside the $6.5 \times 6.9 \AA$ pores parallel to [100] in which the molecules interact through $\pi-\pi$ stacking. Ordered water molecules form infinite hydrogen-bonded chains inside a second pore system $(1.6 \times 5.3 \AA$ free aperture) perpendicular to the $4,4^{\prime}$-bipyridine channels.

\section{Chemical context}

The title compound was obtained in an attempt to reproduce the synthesis of $\left[\mathrm{Cu}\left(\mu-4,4^{\prime}\right.\right.$-bipy $\left.)\left(\mathrm{H}_{2} \mathrm{O}\right)_{2}\left(\mathrm{BF}_{4}\right)_{2}\right] \cdot 4,4^{\prime}$-bipy (Blake et al., 1997). A contamination with $\mathrm{SiF}_{6}{ }^{2-}$ is, however, at the origin of the formation of $\left\{\left[\mathrm{Cu}\left(\mathrm{SiF}_{6}\right)\left(\mathrm{C}_{10} \mathrm{H}_{8} \mathrm{~N}_{2}\right)_{2}\right] \cdot-\right.$ $\left.2 \mathrm{C}_{10} \mathrm{~N}_{2} \mathrm{H}_{8} \cdot 5 \mathrm{H}_{2} \mathrm{O}\right\}_{n}$, whose framework was previously described by Noro et al. $(2000,2002)$. This framework has shown interesting gas adsorption properties in recent years (Burd et al., 2012; Yu et al., 2012; Fan et al., 2013). Several structures based on this porous framework have been published since its discovery [CSD refcodes: GORWUF (Noro et al., 2000), AFEKAX (Noro et al., 2002), HAPKOA (Burd et al., 2012)]. However, these structures which are reported in the tetragonal space group type $P 4 / \mathrm{mmm}$ are disordered: the framework bipyridines are disordered by symmetry whereas solvent molecules are not clearly identified within the pores. In this article, we show that this porous coordination polymer is capable of firmly stabilizing guest entities such as $4,4^{\prime}$-bipyridine and water molecules within its channels. The synthesis conditions thus seem a key factor in producing the ordering of guest molecules within this porous material.

\section{Structural commentary}

The asymmetric unit of the title compound (Fig. 1) contains two copper(II) atoms, both lying on inversion centers; each of these two atoms is coordinated by $\mathrm{N}$ atoms of four symmetrically related 4,4'-bipyridine molecules (with one independent bipyridine for each copper atom), forming slightly distorted two-dimensional square grids parallel to (100). The 
copper(II) atoms are both at the center of elongated octahedra (Table 1).

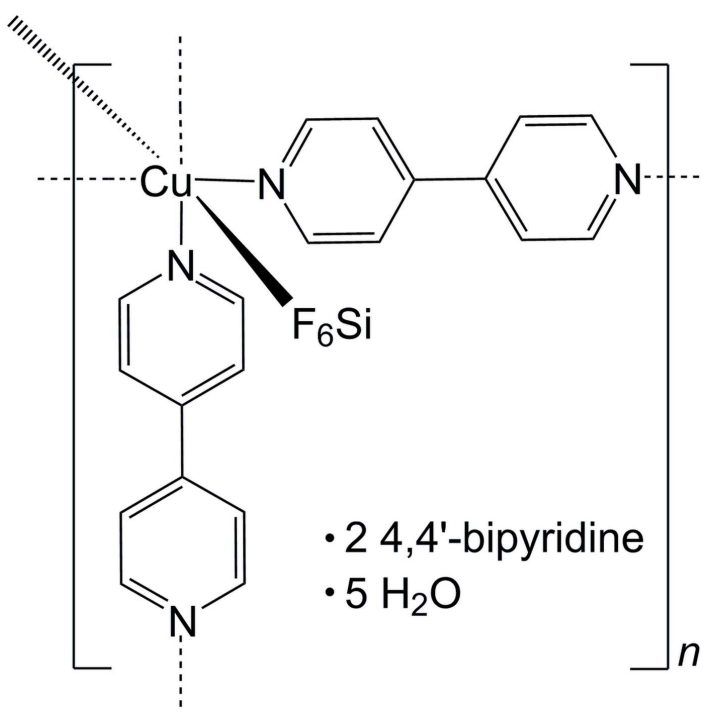

The basal plane is composed of four nitrogen atoms coming from the 4,4'-bipyridine molecules, whereas the apical positions are occupied by fluorine atoms belonging to the $\mathrm{SiF}_{6}{ }^{2-}$ anions pillaring the structure (Fig. 2).

The 2D coordination grids are stacked along the [100] direction through the $\mathrm{SiF}_{6}{ }^{2-}$ anions, leading to a threedimensional coordination polymer, which displays channels having a free aperture of $6.5 \times 6.9 \AA$ parallel to [100] and smaller pores of $1.6 \times 5.3 \AA$ along the [011] direction (as measured in projection in the plane perpendicular to the channels and using van der Waals radii). These interconnected pores are filled with two other 4,4'-bipyridine molecules and five water molecules (Figs. 3 and 4). In comparison, the
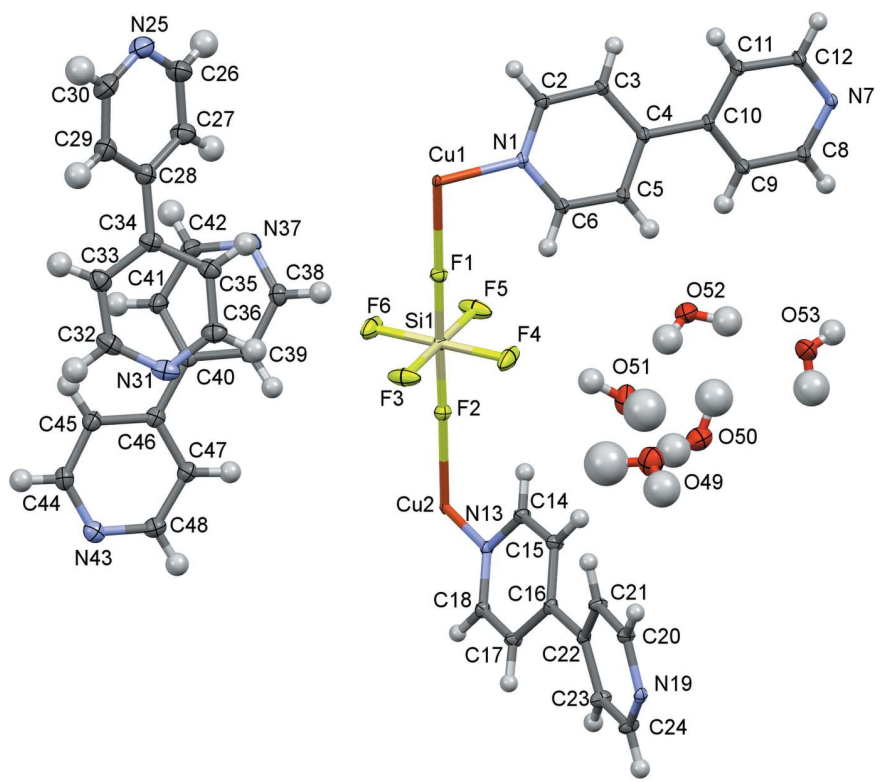

Figure 1

The asymmetric unit of the title compound. Displacement ellipsoids are drawn at the $50 \%$ probability level.
Table 1

Selected geometric parameters $\left(\AA,^{\circ}\right)$.

\begin{tabular}{|c|c|c|c|}
\hline $\mathrm{Cu} 1-\mathrm{N} 1^{\mathrm{i}}$ & $2.0156(11)$ & $\mathrm{Cu} 2-\mathrm{N} 19^{\mathrm{vi}}$ & $2.0494(11)$ \\
\hline $\mathrm{Cu} 1-\mathrm{N} 1$ & $2.0156(11)$ & $\mathrm{Cu} 2-\mathrm{F} 2$ & $2.4109(9)$ \\
\hline $\mathrm{Cu} 1-\mathrm{N} 7^{\mathrm{ii}}$ & $2.0467(11)$ & $\mathrm{Cu} 2-\mathrm{F} 2^{\mathrm{iv}}$ & $2.4109(9)$ \\
\hline $\mathrm{Cu} 1-\mathrm{N} 7^{\mathrm{iii}}$ & $2.0467(11)$ & Si1-F6 & $1.6534(11)$ \\
\hline $\mathrm{Cu} 1-\mathrm{F} 1^{\mathrm{i}}$ & $2.3585(9)$ & Si1-F5 & $1.6806(10)$ \\
\hline $\mathrm{Cu} 1-\mathrm{F} 1$ & $2.3585(9)$ & Si1-F3 & $1.6823(10)$ \\
\hline $\mathrm{Cu} 2-\mathrm{N} 13^{\mathrm{iv}}$ & $2.0170(11)$ & Si1-F4 & $1.6855(11)$ \\
\hline $\mathrm{Cu} 2-\mathrm{N} 13$ & $2.0170(11)$ & Si1-F2 & $1.7120(10)$ \\
\hline $\mathrm{Cu} 2-\mathrm{N} 19^{\mathrm{v}}$ & $2.0494(11)$ & Si1-F1 & $1.7145(10)$ \\
\hline $\mathrm{N} 1{ }^{\mathrm{i}}-\mathrm{Cu} 1-\mathrm{N} 1$ & $180.00(6)$ & $\mathrm{N} 19^{\mathrm{v}}-\mathrm{Cu} 2-\mathrm{F} 2$ & $90.67(4)$ \\
\hline $\mathrm{N} 1^{\mathrm{i}}-\mathrm{Cu} 1-\mathrm{N} 7^{\mathrm{ii}}$ & $91.58(5)$ & $\mathrm{N} 19^{\mathrm{vi}}-\mathrm{Cu} 2-\mathrm{F} 2$ & $89.33(4)$ \\
\hline $\mathrm{N} 1-\mathrm{Cu} 1-\mathrm{N} 7^{\mathrm{ii}}$ & $88.42(5)$ & $\mathrm{N} 13^{\mathrm{iv}}-\mathrm{Cu} 2-\mathrm{F}^{\mathrm{iv}}$ & $89.41(4)$ \\
\hline $\mathrm{N} 1^{\mathrm{i}}-\mathrm{Cu} 1-\mathrm{N} 7^{\mathrm{iii}}$ & $88.42(5)$ & $\mathrm{N} 13-\mathrm{Cu} 2-\mathrm{F}_{2}^{\mathrm{iv}}$ & $90.59(4)$ \\
\hline $\mathrm{N} 1-\mathrm{Cu} 1-\mathrm{N} 7^{\mathrm{iii}}$ & $91.58(5)$ & $\mathrm{N} 19^{\mathrm{v}}-\mathrm{Cu} 2-\mathrm{F} 2^{\mathrm{iv}}$ & $89.33(4)$ \\
\hline $\mathrm{N} 7^{\mathrm{ii}}-\mathrm{Cu} 1-\mathrm{N} 7^{\mathrm{iii}}$ & 180.0 & $\mathrm{~N} 19^{\mathrm{vi}}-\mathrm{Cu} 2-\mathrm{F}^{\mathrm{iv}}$ & $90.67(4)$ \\
\hline $\mathrm{N} 1^{\mathrm{i}}-\mathrm{Cu} 1-\mathrm{F} 1^{\mathrm{i}}$ & $90.65(4)$ & $\mathrm{F} 2-\mathrm{Cu} 2-\mathrm{F} 2^{\mathrm{iv}}$ & 180.0 \\
\hline $\mathrm{N} 1-\mathrm{Cu} 1-\mathrm{F} 1^{\mathrm{i}}$ & $89.35(4)$ & F6-Si1-F5 & $90.80(6)$ \\
\hline $\mathrm{N} 7^{\mathrm{ii}}-\mathrm{Cu} 1-\mathrm{F} 1^{\mathrm{i}}$ & $89.60(4)$ & F6-Si1-F3 & $89.64(6)$ \\
\hline $\mathrm{N} 7^{\mathrm{iii}}-\mathrm{Cu} 1-\mathrm{F} 1^{\mathrm{i}}$ & $90.40(4)$ & $\mathrm{F} 5-\mathrm{Si} 1-\mathrm{F} 3$ & $179.55(6)$ \\
\hline $\mathrm{N} 1^{\mathrm{i}}-\mathrm{Cu} 1-\mathrm{F} 1$ & $89.35(4)$ & $\mathrm{F} 6-\mathrm{Si} 1-\mathrm{F} 4$ & $178.87(6)$ \\
\hline $\mathrm{N} 1-\mathrm{Cu} 1-\mathrm{F} 1$ & $90.65(4)$ & F5-Si1-F4 & $90.12(6)$ \\
\hline $\mathrm{N} 7^{\mathrm{ii}}-\mathrm{Cu} 1-\mathrm{F} 1$ & $90.40(4)$ & $\mathrm{F} 3-\mathrm{Si} 1-\mathrm{F} 4$ & $89.44(6)$ \\
\hline $\mathrm{N} 7^{\mathrm{iii}}-\mathrm{Cu} 1-\mathrm{F} 1$ & $89.60(4)$ & $\mathrm{F} 6-\mathrm{Si} 1-\mathrm{F} 2$ & $91.01(4)$ \\
\hline $\mathrm{F}^{\mathrm{i}}-\mathrm{Cu} 1-\mathrm{F} 1$ & 180.0 & $\mathrm{~F} 5-\mathrm{Si} 1-\mathrm{F} 2$ & $89.67(5)$ \\
\hline $\mathrm{N} 13^{\mathrm{iv}}-\mathrm{Cu} 2-\mathrm{N} 13$ & 180.0 & $\mathrm{~F} 3-\mathrm{Si} 1-\mathrm{F} 2$ & $90.41(5)$ \\
\hline $\mathrm{N} 13^{\mathrm{iv}}-\mathrm{Cu} 2-\mathrm{N} 19^{\mathrm{v}}$ & $90.73(5)$ & $\mathrm{F} 4-\mathrm{Si} 1-\mathrm{F} 2$ & $89.64(4)$ \\
\hline $\mathrm{N} 13-\mathrm{Cu} 2-\mathrm{N} 19^{\mathrm{v}}$ & $89.27(5)$ & F6-Si1-F1 & $90.89(4)$ \\
\hline $\mathrm{N} 13^{\mathrm{iv}}-\mathrm{Cu} 2-\mathrm{N} 19^{\mathrm{vi}}$ & $89.27(5)$ & F5-Si1-F1 & $90.35(5)$ \\
\hline $\mathrm{N} 13-\mathrm{Cu} 2-\mathrm{N} 19^{\mathrm{vi}}$ & $90.73(5)$ & $\mathrm{F} 3-\mathrm{Si} 1-\mathrm{F} 1$ & $89.56(5)$ \\
\hline $\mathrm{N} 19^{\mathrm{v}}-\mathrm{Cu} 2-\mathrm{N} 19^{\mathrm{vi}}$ & 180.0 & $\mathrm{~F} 4-\mathrm{Si} 1-\mathrm{F} 1$ & $88.45(4)$ \\
\hline $\mathrm{N} 13^{\mathrm{iv}}-\mathrm{Cu} 2-\mathrm{F} 2$ & $90.59(4)$ & $\mathrm{F} 2-\mathrm{Si} 1-\mathrm{F} 1$ & $178.09(4)$ \\
\hline $\mathrm{N} 13-\mathrm{Cu} 2-\mathrm{F} 2$ & $89.41(4)$ & $\mathrm{Si} 1-\mathrm{F} 1-\mathrm{Cu} 1$ & $178.36(5)$ \\
\hline
\end{tabular}

Symmetry codes: (i) $-x,-y+1,-z+1$; (ii) $x,-y+\frac{1}{2}, z-\frac{1}{2}$; (iii) $-x, y+\frac{1}{2},-z+\frac{3}{2}$; (iv) $-x+1,-y+1,-z+1 ;$ (v) $x,-y+\frac{1}{2}, z+\frac{1}{2} ;(\mathrm{vi})-x+1, y+\frac{1}{2},-z+\frac{1}{2}$.

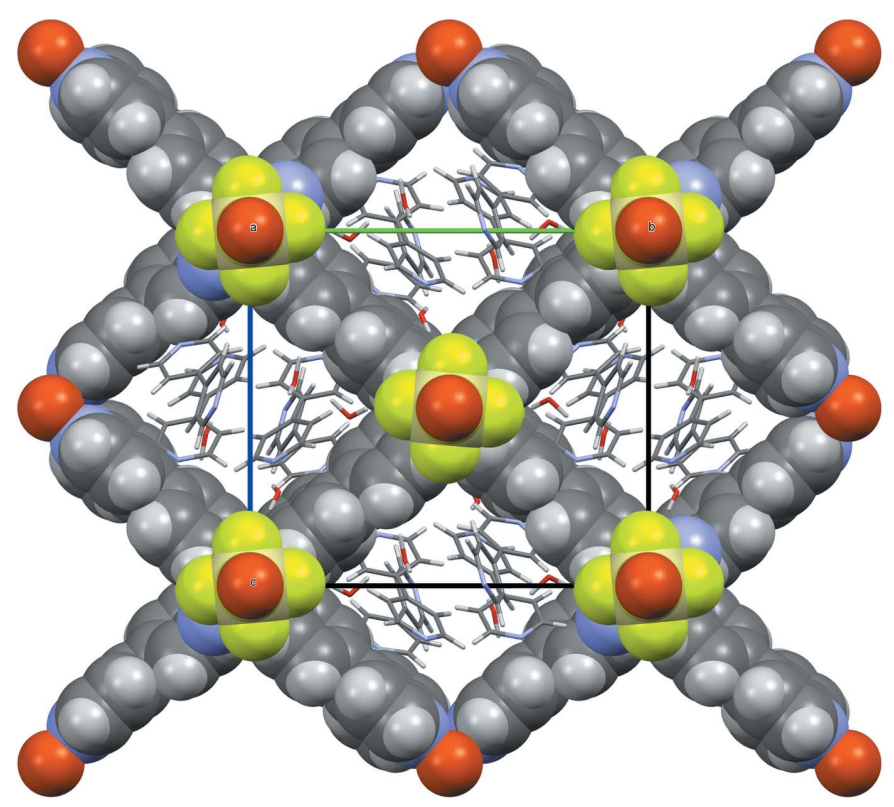

Figure 2

View along [011]; the atoms belonging to the framework are shown as space-filling, whereas the molecules adsorbed inside the pores are shown as capped sticks. 


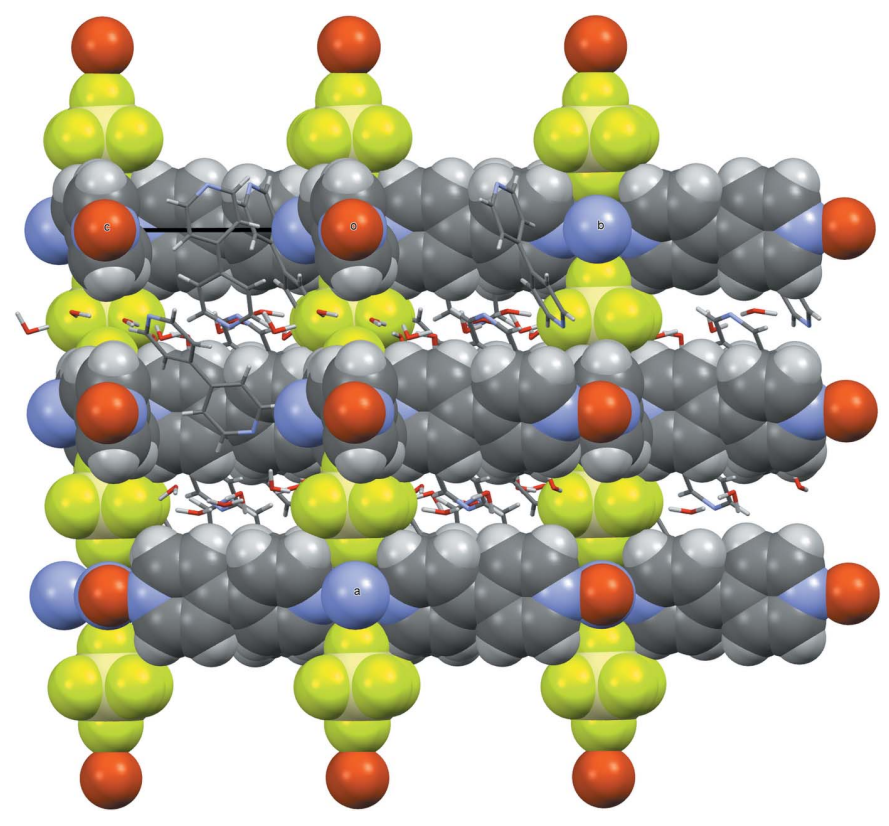

Figure 3

View along [100]; the atoms belonging to the framework are shown as space-filling, whereas the molecules inside the pores are shown as capped sticks.

previously reported structures with this framework are described in the $P 4 / \mathrm{mmm}$ space group type, implying a squared $\mathrm{Cu}$ grid and channels; here, the $\mathrm{Cu}-\mathrm{Cu}-\mathrm{Cu}$ grid angle

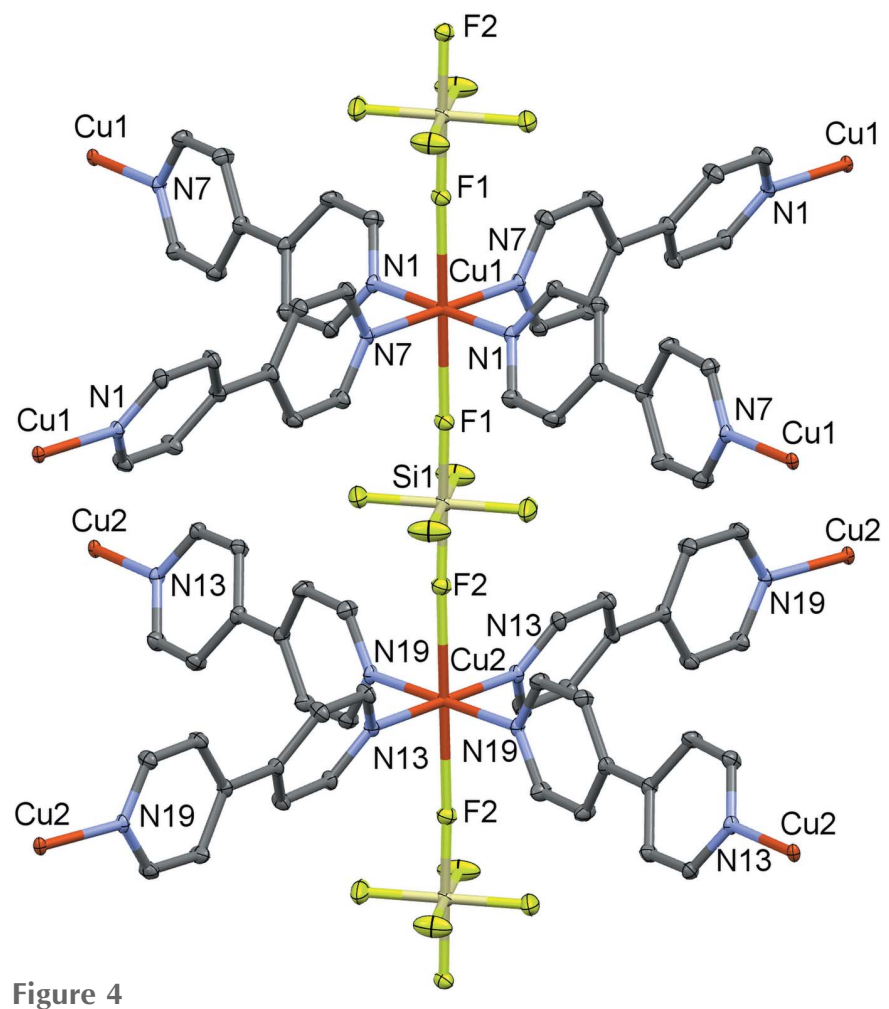

Figure 4

$O R T E P$-style plot of the title compound showing the coordination about the two inequivalent copper atoms. Hydrogen atoms, adsorbed 4,4'bipyridine and water molecules are omitted for clarity. Displacement ellipsoids are drawn at the $50 \%$ probability level.
Table 2

Hydrogen-bond geometry $\left(\AA{ }^{\circ}\right)$.

\begin{tabular}{|c|c|c|c|c|}
\hline$D-\mathrm{H} \cdots A$ & $D-\mathrm{H}$ & $\mathrm{H} \cdots A$ & $D \cdots A$ & $D-\mathrm{H} \cdots A$ \\
\hline $\mathrm{C} 17-\mathrm{H} 17 \cdots \mathrm{N} 31^{\mathrm{vii}}$ & 0.95 & 2.34 & $3.2849(19)$ & 170 \\
\hline $\mathrm{C} 20-\mathrm{H} 20 \cdots \mathrm{F} 2^{\mathrm{ii}}$ & 0.95 & 2.32 & $3.0341(16)$ & 131 \\
\hline $\mathrm{C} 20-\mathrm{H} 20 \cdots \mathrm{F} 5^{\mathrm{ii}}$ & 0.95 & 2.53 & 3.4305 (17) & 159 \\
\hline $\mathrm{C} 18-\mathrm{H} 18 \cdots \mathrm{F}^{\text {iv }}$ & 0.95 & 2.52 & 3.1085 (16) & 120 \\
\hline $\mathrm{C} 9-\mathrm{H} 9 \cdots \mathrm{O} 52$ & 0.95 & 2.50 & $3.4223(18)$ & 163 \\
\hline $\mathrm{C} 3-\mathrm{H} 3 \cdots \mathrm{N} 37^{\mathrm{viii}}$ & 0.95 & 2.54 & $3.4803(19)$ & 173 \\
\hline $\mathrm{C} 21-\mathrm{H} 21 \cdots \mathrm{O} 52^{\mathrm{ii}}$ & 0.95 & 2.56 & $3.4294(18)$ & 152 \\
\hline 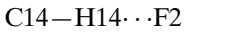 & 0.95 & 2.43 & $3.0497(16)$ & 123 \\
\hline $\mathrm{C} 14-\mathrm{H} 14 \cdots \mathrm{F} 4$ & 0.95 & 2.55 & $3.4519(17)$ & 158 \\
\hline $\mathrm{C} 8-\mathrm{H} 8 \cdots \mathrm{F} 1^{\mathrm{v}}$ & 0.95 & 2.33 & $3.0205(16)$ & 129 \\
\hline $\mathrm{C} 8-\mathrm{H} 8 \cdots \mathrm{F} 4^{\mathrm{v}}$ & 0.95 & 2.51 & $3.3524(18)$ & 148 \\
\hline $\mathrm{C} 5-\mathrm{H} 5 \cdots \mathrm{O} 52$ & 0.95 & 2.43 & $3.2916(18)$ & 151 \\
\hline $\mathrm{C} 24-\mathrm{H} 24 \cdots \mathrm{F} 2^{\mathrm{vii}}$ & 0.95 & 2.29 & $2.9997(16)$ & 131 \\
\hline $\mathrm{C} 24-\mathrm{H} 24 \cdots \mathrm{F}^{\mathrm{vii}}$ & 0.95 & 2.55 & 3.4609 (17) & 161 \\
\hline $\mathrm{C} 11-\mathrm{H} 11 \cdots \mathrm{N} 37^{\mathrm{viii}}$ & 0.95 & 2.61 & $3.4358(19)$ & 145 \\
\hline $\mathrm{C} 12-\mathrm{H} 12 \cdots \mathrm{F} 1^{\mathrm{viii}}$ & 0.95 & 2.26 & $2.9662(16)$ & 131 \\
\hline $\mathrm{C} 12-\mathrm{H} 12 \cdots \mathrm{F}{ }^{\mathrm{viii}}$ & 0.95 & 2.49 & $3.4040(16)$ & 161 \\
\hline $\mathrm{C} 2-\mathrm{H} 2 \cdots \mathrm{F} 1^{\mathrm{i}}$ & 0.95 & 2.52 & $3.0708(16)$ & 117 \\
\hline $\mathrm{C} 2-\mathrm{H} 2 \cdots \mathrm{F} 3^{\mathrm{i}}$ & 0.95 & 2.45 & 3.3364 (17) & 156 \\
\hline $\mathrm{C} 45-\mathrm{H} 45 \cdots \mathrm{O} 50^{\mathrm{ix}}$ & 0.95 & 2.44 & $3.376(2)$ & 167 \\
\hline 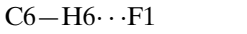 & 0.95 & 2.46 & $3.0691(16)$ & 122 \\
\hline $\mathrm{C} 6-\mathrm{H} 6 \cdots \mathrm{F} 4$ & 0.95 & 2.60 & $3.5313(17)$ & 166 \\
\hline 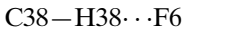 & 0.95 & 2.41 & $3.1240(19)$ & 132 \\
\hline $\mathrm{C} 48-\mathrm{H} 48 \cdots \mathrm{O} 51^{\mathrm{iv}}$ & 0.95 & 2.50 & $3.330(2)$ & 146 \\
\hline $\mathrm{O} 53-\mathrm{H} 53 B \cdots \mathrm{F}^{\mathrm{v}}$ & $0.78(2)$ & $1.96(3)$ & $2.7228(17)$ & $166(2)$ \\
\hline $\mathrm{O} 52-\mathrm{H} 52 B \cdots \mathrm{O} 53$ & $0.90(3)$ & $1.83(3)$ & $2.7227(18)$ & $170(2)$ \\
\hline $\mathrm{O} 53-\mathrm{H} 53 A \cdots \mathrm{N} 43^{\text {iv }}$ & $0.85(3)$ & $2.05(3)$ & $2.8666(19)$ & $161(3)$ \\
\hline $\mathrm{O} 52-\mathrm{H} 52 A \cdots \mathrm{O} 51$ & $0.90(3)$ & $1.84(3)$ & $2.726(2)$ & $172(2)$ \\
\hline $\mathrm{O} 50-\mathrm{H} 50 A \cdots \mathrm{O} 49$ & $1.05(3)$ & $1.72(3)$ & $2.741(2)$ & $163(2)$ \\
\hline $\mathrm{O} 49-\mathrm{H} 49 B \cdots \mathrm{F} 5^{\mathrm{ii}}$ & $0.96(3)$ & $1.82(3)$ & $2.7716(18)$ & $173(3)$ \\
\hline $\mathrm{O} 50-\mathrm{H} 50 B \cdots \mathrm{N} 25^{\mathrm{i}}$ & $0.96(3)$ & $1.86(3)$ & $2.816(2)$ & $173(3)$ \\
\hline $\mathrm{O} 49-\mathrm{H} 49 A \cdots \mathrm{O} 52^{\mathrm{ii}}$ & $1.00(4)$ & $1.81(4)$ & $2.798(2)$ & $169(3)$ \\
\hline $\mathrm{O} 51-\mathrm{H} 51 B \cdots \mathrm{F} 4$ & $0.91(3)$ & $1.88(3)$ & $2.7029(18)$ & $149(2)$ \\
\hline $\mathrm{O} 51-\mathrm{H} 51 A \cdots \mathrm{O} 50$ & $1.00(4)$ & $1.76(4)$ & $2.719(2)$ & $158(3)$ \\
\hline
\end{tabular}

significantly deviates from $90^{\circ}\left(96.62^{\circ}\right)$ and this may be related to the fact that, in the present compound, guest molecules fill the pores and interact significantly with the framework atoms (see below).

The $\mathrm{Si}-\mathrm{F}$ bond lengths in $\mathrm{SiF}_{6}{ }^{2-}$ show some variations (Table 1), ranging from 1.6534 (11) to 1.7145 (10) $\AA$. The two longest bond lengths are associated with opposite fluorine atoms bounded to a $\mathrm{Cu}^{\mathrm{II}}$ metal atom. Among the four remaining fluorine atoms, three of them form short hydrogen bonds with water molecules and display longer bond lengths than the last one, which only forms a weaker hydrogenbonding interaction with a 4,4'-bipyridine molecule (Table 2). A search for $\mathrm{SiF}_{6}{ }^{2-}$ anions within the Cambridge Structural Database (CSD Version 5.36; Groom et al., 2016) leads to 241 hits (using options 'not disordered'" and 'no errors'); the reported $\mathrm{Si}-\mathrm{F}$ bond lengths range from 1.577 to $1.748 \AA$ with a mean of $1.684 \AA$ and a standard deviation of $0.022 \AA$.

\section{Supramolecular features}

Four of the five water molecules (O49 to O52) form infinite $C_{4}^{4}(2)$ chains running in the [001] direction throughout the pores (Fig. 5). The fifth water molecule (O53) interacts with 


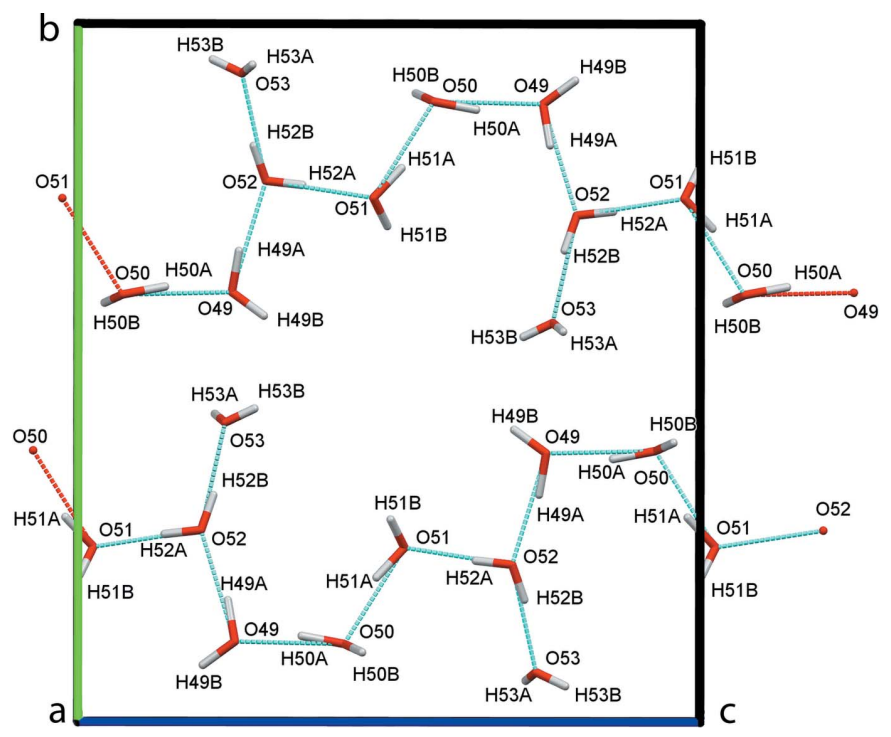

Figure 5

Hydrogen-bonding pattern of solvate water molecules, forming infinite chains along the [001] direction. All other atoms apart from those of the water molecules are omitted for clarity.

these chains and several hydrogen bonds anchor these water molecules to the coordination polymer framework (Table 2). The 4,4'-bipyridine molecules filling the [100] channels form chains through $\pi-\pi$ stacking (Fig. 6; Table 3), and are connected to three of the water molecules (O50, O51 and

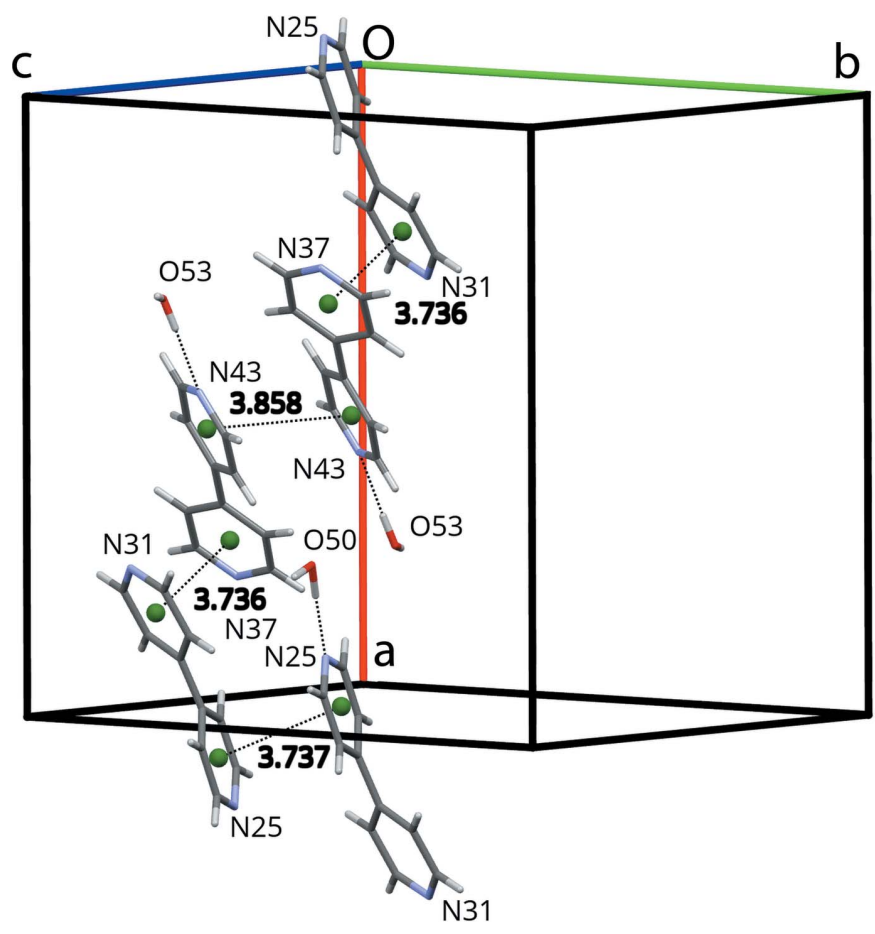

Figure 6

$\pi-\pi$ stacking pattern of the solvent 4,4'-bipyridine molecules in the [100] pores. All other atoms (except water molecules hydrogen-bonded to $\mathrm{N}$ atoms of these bipyridines) are omitted for clarity. Pyridyl centroids are displayed as green spheres.
Table 3

Geometrical parameters $\left(\AA{ }^{\circ}\right)$ for the $\pi-\pi$ stacking of the $4,4^{\prime}$-bipyridine molecules within the pores.

$C g(I)$ is the centroid of the atoms defining plane $I: C g(\mathrm{~N} 25)=\mathrm{N} 25 / \mathrm{C} 26-\mathrm{C} 30$; $C g(\mathrm{~N} 31)=\mathrm{N} 31 / \mathrm{C} 32-\mathrm{C} 36 ; C g(\mathrm{~N} 43)=\mathrm{N} 43 / \mathrm{C} 44-7-\mathrm{C} 48$ and $C g(\mathrm{~N} 37)=\mathrm{N} 37 /$ C38-C42. $d_{C g-C g}$ is the distance between $C g(I)$ and $C g(J) . \alpha$ is the dihedral angle between planes $I$ and $J$. $\beta$ is the angle between the $C g(I) \rightarrow C g(J)$ vector and the normal to plane $I . \gamma$ is the angle between the $C g(I) \rightarrow C g(J)$ vector and the normal to plane $J$.

\begin{tabular}{llllll}
\hline$C g(I)$ & $C g(J)$ & $d_{C g-C g}$ & $\alpha$ & $\beta$ & $\gamma$ \\
\hline N25 & N25 & $3.7374(10)$ & $0.02(9)$ & 9.2 & 9.2 \\
N31 & N37 $^{\text {ii }}$ & $3.7358(9)$ & $20.79(8)$ & 21.4 & 13.1 \\
N43 & N43 $^{\text {iii }}$ & $3.8576(9)$ & $0.00(7)$ & 23.8 & 23.8 \\
\hline
\end{tabular}

Symmetry codes: (i) $-x, 2-y, 1-z$; (ii) $x, y, z$; (iii) $1-x, 2-y, 1-z$.

O53) and framework fluorine and aromatic hydrogen atoms by hydrogen bonds (Table 2); these intermolecular interactions induce different dihedral angles within the two symmetry-independent 4,4'-bipyridine molecules bipy $_{(\mathrm{N} 25-\mathrm{N} 31)}: 45.29(7)^{\circ}$; bipy $\left._{(\mathrm{N} 37-\mathrm{N} 43)}: 30.31(7)^{\circ}\right]$. Whereas the $4,4^{\prime}$-bipyridine molecules belonging to the coordination network are rather rigid between the metal atoms [average $U_{\text {eq }}=0.014(2) \AA^{2}$ as calculated on C,N atoms], the adsorbed 4,4'-bipyridine molecules display significantly larger atomic displacement parameters $\left[U_{\mathrm{eq}}=0.025(5) \AA^{2}\right]$.

\section{Database survey}

A survey was performed in the Cambridge Structural Database (CSD Version 5.36; Groom et al., 2016). Beside the structures corresponding to the bare or hydrated $\left[\mathrm{Cu}\left(\mathrm{SiF}_{6}\right)\left(\mathrm{C}_{10} \mathrm{H}_{8} \mathrm{~N}_{2}\right)_{2}\right]_{n}$ coordination polymer framework [CSD refcodes: GORWUF (Noro et al., 2000), AFEKAX (Noro et al., 2002), HAPKOA (Burd et al., 2012)], several structures related to the title compound have been described. In particular, Noro et al. showed that the hydrated form of the title compound $\left\{\left[\mathrm{Cu}\left(\mathrm{SiF}_{6}\right)\left(4,4^{\prime}-\text { bpy }\right)_{2}\right] \cdot 8 \mathrm{H}_{2} \mathrm{O}\right\}_{n}$ undergoes a structural conversion when immersed in water, leading to an interpenetrated network where $\mathrm{SiF}_{6}{ }^{2-}$ anions are shifted out of the coordination sphere of copper ions and are replaced by water molecules [CSD refcodes: AFEHOI (Noro et al., 2002); JEZRUB (Gable et al., 1990)]. When copper is replaced by zinc, an isostructural compound is obtained [CSD refcodes: WONZIJ (Lin et al., 2009); ZESFUY (Subramanian \& Zaworotko, 1995)].

\section{Synthesis and crystallization}

An aqueous solution $\left(5 \mathrm{~cm}^{3}\right)$ of hydrated copper(II) tetrafluoridoborate $(47.43 \mathrm{mg}, 0.2 \mathrm{mmol})$ was added to a refluxing acetonitrile solution $\left(5 \mathrm{~cm}^{3}\right)$ of $4,4^{\prime}$-bipyridine $(62.48 \mathrm{mg}$, $0.4 \mathrm{mmol})$. After filtration, $\mathrm{Et}_{2} \mathrm{O}$ vapor was diffused into the mother liquor for seven days, and then the solvent was allowed to evaporate very slowly. A mixture of blue and violet crystals was obtained; whereas the diffraction spots of the blue crystals could not be properly indexed, the violet crystals were of very good quality and led to the structure reported on herein. 


\section{Refinement}

Crystal data, data collection and structure refinement details are summarized in Table 4. All hydrogen atoms of water molecules were freely refined in an isotropic approximation. Aromatic hydrogen atoms were refined with riding coordinates and $U_{\text {iso }}(\mathrm{H})=1.2 U_{\text {iso }}(\mathrm{C})$.

\section{Acknowledgements}

The Service Commun de Diffraction des Rayons X - Lorraine University is thanked for providing access to crystallographic facilities.

\section{References}

Blake, A. J., Hill, S. J., Hubberstey, P. \& Li, W.-S. (1997). J. Chem. Soc. Dalton Trans. pp. 913-914.

Burd, S. D., Ma, S., Perman, J. A., Sikora, B. J., Snurr, R. Q., Thallapally, P. K., Tian, J., Wojtas, L. \& Zaworotko, M. J. (2012). J. Am. Chem. Soc. 134, 3663-3666.

Clark, R. C. \& Reid, J. S. (1995). Acta Cryst. A51, 887-897.

Fan, S., Sun, F., Xie, J., Guo, J., Zhang, L., Wang, C., Pan, Q. \& Zhu, G. (2013). J. Mater. Chem. A, 1, 11438-11442.

Gable, R. W., Hoskins, B. F. \& Robson, R. (1990). J. Chem. Soc. Chem. Commun. pp. 1677-1678.

Groom, C. R., Bruno, I. J., Lightfoot, M. P. \& Ward, S. C. (2016). Acta Cryst. B72, 171-179.

Lin, M.-J., Jouaiti, A., Kyritsakas, N. \& Hosseini, M. W. (2009). CrystEngComm, 11, 189-191.

Macrae, C. F., Edgington, P. R., McCabe, P., Pidcock, E., Shields, G. P., Taylor, R., Towler, M. \& van de Streek, J. (2006). J. Appl. Cryst. 39, 453-457.

Noro, S., Kitagawa, S., Kondo, M. \& Seki, K. (2000). Angew. Chem. Int. Ed. 39, 2081-2084.

Noro, S., Kitaura, R., Kondo, M., Kitagawa, S., Ishii, T., Matsuzaka, H. \& Yamashita, M. (2002). J. Am. Chem. Soc. 124, 2568-2583.

Rigaku Oxford Diffraction (2015). Crys Alis PRO. Rigaku Corporation, Tokyo, Japan.

Sheldrick, G. M. (2015a). Acta Cryst. A71, 3-8.

Sheldrick, G. M. (2015b). Acta Cryst. C71, 3-8.

Subramanian, S. \& Zaworotko, M. J. (1995). Angew. Chem. Int. Ed. Engl. 34, 2127-2129.
Table 4

Experimental details.

\begin{tabular}{|c|c|}
\hline \multicolumn{2}{|l|}{ Crystal data } \\
\hline Chemical formula & $\begin{array}{l}{\left[\mathrm{Cu}\left(\mathrm{SiF}_{6}\right)\left(\mathrm{C}_{10} \mathrm{H}_{8} \mathrm{~N}_{2}\right)_{2}\right] \cdot 2 \mathrm{C}_{10} \mathrm{H}_{8} \mathrm{~N}_{2} \cdot-} \\
\quad 5 \mathrm{H}_{2} \mathrm{O}\end{array}$ \\
\hline$M_{\mathrm{r}}$ & 920.44 \\
\hline Crystal system, space group & Monoclinic, $P 2_{1} / c$ \\
\hline Temperature $(\mathrm{K})$ & 110 \\
\hline$a, b, c(\AA)$ & $\begin{array}{l}16.3875(2), 16.6136(2), \\
14.7959(2)\end{array}$ \\
\hline$\beta\left({ }^{\circ}\right)$ & $90.654(1)$ \\
\hline$V\left(\AA^{3}\right)$ & $4028.00(9)$ \\
\hline$Z$ & 4 \\
\hline Radiation type & $\mathrm{Cu} K \alpha$ \\
\hline$\mu\left(\mathrm{mm}^{-1}\right)$ & 1.78 \\
\hline Crystal size $(\mathrm{mm})$ & $0.25 \times 0.16 \times 0.14$ \\
\hline \multicolumn{2}{|l|}{ Data collection } \\
\hline Diffractometer & $\begin{array}{l}\text { Rigaku Oxford Diffraction Super- } \\
\text { Nova (Cu) X-ray Source }\end{array}$ \\
\hline Absorption correction & $\begin{array}{l}\text { Analytical [CrysAlis PRO (Rigaku } \\
\text { Oxford Diffraction, 2015) based } \\
\text { on expressions derived by Clark } \\
\text { \& Reid (1995)] }\end{array}$ \\
\hline$T_{\min }, T_{\max }$ & $0.708,0.824$ \\
\hline $\begin{array}{l}\text { No. of measured, independent and } \\
\text { observed }[I>2 \sigma(I)] \text { reflections }\end{array}$ & $68271,8435,7846$ \\
\hline$R_{\text {int }}$ & 0.029 \\
\hline$(\sin \theta / \lambda)_{\max }\left(\AA^{-1}\right)$ & 0.630 \\
\hline \multicolumn{2}{|l|}{ Refinement } \\
\hline$R\left[F^{2}>2 \sigma\left(F^{2}\right)\right], w R\left(F^{2}\right), S$ & $0.036,0.110,1.06$ \\
\hline No. of reflections & 8435 \\
\hline No. of parameters & 593 \\
\hline $\mathrm{H}$-atom treatment & $\begin{array}{l}\mathrm{H} \text { atoms treated by a mixture of } \\
\text { independent and constrained } \\
\text { refinement }\end{array}$ \\
\hline$\Delta \rho_{\max }, \Delta \rho_{\min }\left(\mathrm{e} \AA^{-3}\right)$ & $0.47,-0.50$ \\
\hline
\end{tabular}

Computer programs: CrysAlis PRO (Rigaku Oxford Diffraction, 2015), SHELXT (Sheldrick, 2015a), SHELXL (Sheldrick, 2015b), Mercury (Macrae et al., 2006) and publCIF (Westrip, 2010).

Westrip, S. P. (2010). J. Appl. Cryst. 43, 920-925.

Yu, Q., Yang, J., Zhao, Q., Dong, J. \& Li, J. (2012). J. Coord. Chem. 65, $1645-1654$. 


\section{supporting information}

Acta Cryst. (2016). E72, 1654-1658 [https://doi.org/10.1107/S2056989016016686]

\section{Channels with ordered water and bipyridine molecules in the porous coordination polymer $\left\{\left[\mathrm{Cu}\left(\mathrm{SiF}_{6}\right)\left(\mathrm{C}_{10} \mathrm{H}_{8} \mathrm{~N}_{2}\right)_{2}\right] \cdot 2 \mathrm{C}_{10} \mathrm{~N}_{2} \mathrm{H}_{8} \cdot 5 \mathrm{H}_{2} \mathrm{O}\right\}_{n}$}

\section{Emmanuel Aubert, Abdelatif Doudouh, Paola Peluso and Victor Mamane}

Computing details

Data collection: CrysAlis PRO (Rigaku Oxford Diffraction, 2015); cell refinement: CrysAlis PRO (Rigaku Oxford Diffraction, 2015); data reduction: CrysAlis PRO (Rigaku Oxford Diffraction, 2015); program(s) used to solve structure: SHELXT (Sheldrick, 2015a); program(s) used to refine structure: SHELXL (Sheldrick, 2015b); molecular graphics: Mercury (Macrae et al., 2006); software used to prepare material for publication: publCIF (Westrip, 2010).

Poly[[bis $\left(\mu_{2}-4,4^{\prime}\right.$-bipyridine) $\left(\mu_{2}\right.$-hexafluoridosilicato)copper(II)] 4,4'-bipyridine disolvate pentahydrate]

\section{Crystal data}

$\left[\mathrm{Cu}\left(\mathrm{SiF}_{6}\right)\left(\mathrm{C}_{10} \mathrm{H}_{8} \mathrm{~N}_{2}\right)_{2}\right] \cdot 2 \mathrm{C}_{10} \mathrm{H}_{8} \mathrm{~N}_{2} \cdot 5 \mathrm{H}_{2} \mathrm{O}$

$M_{r}=920.44$

Monoclinic, $P 2_{1} / c$

$a=16.3875(2) \AA$

$b=16.6136(2) \AA$

$c=14.7959(2) \AA$

$\beta=90.654(1)^{\circ}$

$V=4028.00(9) \AA^{3}$

$Z=4$

\section{Data collection}

Rigaku Oxford Diffraction SuperNova $(\mathrm{Cu}) \mathrm{X}$ ray Source diffractometer

Radiation source: micro-focus sealed X-ray tube $\omega$ scans

Absorption correction: analytical [CrysAlis PRO (Rigaku Oxford Diffraction, 2015)]; analytical numeric absorption correction using a multi-faceted crystal model based on expressions derived by Clark \& Reid (1995)

\section{Refinement}

Refinement on $F^{2}$

Least-squares matrix: full

$R\left[F^{2}>2 \sigma\left(F^{2}\right)\right]=0.036$

$w R\left(F^{2}\right)=0.110$

$S=1.06$

8435 reflections

593 parameters
$F(000)=1900$

$D_{\mathrm{x}}=1.518 \mathrm{Mg} \mathrm{m}^{-3}$

$\mathrm{Cu} K \alpha$ radiation, $\lambda=1.54184 \AA$

Cell parameters from 32121 reflections

$\theta=3.7-76.5^{\circ}$

$\mu=1.78 \mathrm{~mm}^{-1}$

$T=110 \mathrm{~K}$

Prism, violet

$0.25 \times 0.16 \times 0.14 \mathrm{~mm}$

$T_{\min }=0.708, T_{\max }=0.824$

68271 measured reflections

8435 independent reflections

7846 reflections with $I>2 \sigma(I)$

$R_{\text {int }}=0.029$

$\theta_{\max }=76.2^{\circ}, \theta_{\min }=3.8^{\circ}$

$h=-20 \rightarrow 20$

$k=0 \rightarrow 20$

$l=0 \rightarrow 18$

0 restraints

Primary atom site location: iterative Hydrogen site location: difference Fourier map

$\mathrm{H}$ atoms treated by a mixture of independent and constrained refinement

$w=1 /\left[\sigma^{2}\left(F_{\mathrm{o}}^{2}\right)+(0.0562 P)^{2}+2.6502 P\right]$ where $P=\left(F_{\mathrm{o}}^{2}+2 F_{\mathrm{c}}{ }^{2}\right) / 3$ 
$(\Delta / \sigma)_{\max }=0.002$

$\Delta \rho_{\min }=-0.50$ e $\AA^{-3}$

$\Delta \rho_{\max }=0.47 \mathrm{e} \AA^{-3}$

Special details

Geometry. All esds (except the esd in the dihedral angle between two 1.s. planes) are estimated using the full covariance matrix. The cell esds are taken into account individually in the estimation of esds in distances, angles and torsion angles; correlations between esds in cell parameters are only used when they are defined by crystal symmetry. An approximate (isotropic) treatment of cell esds is used for estimating esds involving l.s. planes.

Fractional atomic coordinates and isotropic or equivalent isotropic displacement parameters $\left(\AA^{2}\right)$

\begin{tabular}{|c|c|c|c|c|}
\hline & $x$ & $y$ & $z$ & $U_{\text {iso }} * / U_{\text {eq }}$ \\
\hline $\mathrm{Cu} 1$ & 0.0000 & 0.5000 & 0.5000 & $0.00740(9)$ \\
\hline $\mathrm{Cu} 2$ & 0.5000 & 0.5000 & 0.5000 & $0.00808(9)$ \\
\hline Sil & $0.24851(2)$ & $0.50181(2)$ & $0.49951(2)$ & $0.01085(11)$ \\
\hline F1 & $0.14392(6)$ & $0.50149(4)$ & $0.50157(6)$ & $0.01423(19)$ \\
\hline $\mathrm{F} 2$ & $0.35288(6)$ & $0.49872(4)$ & $0.49705(6)$ & 0.01514 (19) \\
\hline F5 & $0.25214(6)$ & $0.49217(7)$ & $0.61255(7)$ & $0.0327(3)$ \\
\hline F6 & $0.25001(5)$ & $0.60099(6)$ & $0.50857(8)$ & $0.0313(3)$ \\
\hline F3 & $0.24470(6)$ & $0.51068(7)$ & $0.38628(7)$ & $0.0305(2)$ \\
\hline F4 & $0.24575(5)$ & $0.40086(6)$ & $0.48849(9)$ & $0.0356(3)$ \\
\hline N1 & $-0.00035(7)$ & $0.41543(7)$ & $0.59766(8)$ & $0.0104(2)$ \\
\hline O52 & $0.24669(7)$ & $0.22533(8)$ & $0.69796(10)$ & 0.0304 (3) \\
\hline N7 & $-0.00003(7)$ & $0.09067(7)$ & $0.90635(7)$ & $0.0104(2)$ \\
\hline N19 & $0.50157(7)$ & $0.08206(7)$ & $0.10342(7)$ & $0.0103(2)$ \\
\hline N13 & $0.50009(7)$ & $0.40834(7)$ & $0.41061(7)$ & $0.0102(2)$ \\
\hline $\mathrm{O} 53$ & $0.29213(8)$ & $0.07136(8)$ & $0.73649(9)$ & $0.0319(3)$ \\
\hline $\mathrm{O} 50$ & $0.27901(8)$ & $0.11159(8)$ & $0.42810(9)$ & $0.0326(3)$ \\
\hline O51 & $0.29696(9)$ & $0.24956(9)$ & $0.52512(10)$ & $0.0374(3)$ \\
\hline N43 & $0.55301(8)$ & $0.94138(8)$ & $0.35053(9)$ & $0.0224(3)$ \\
\hline O49 & $0.22422(9)$ & $0.11500(9)$ & $0.25239(9)$ & $0.0375(3)$ \\
\hline N37 & $0.23466(8)$ & $0.82015(8)$ & $0.64527(10)$ & $0.0244(3)$ \\
\hline N31 & $0.26526(8)$ & $0.82439(10)$ & $0.32164(10)$ & $0.0281(3)$ \\
\hline $\mathrm{C} 16$ & $0.50172(8)$ & $0.27683(8)$ & $0.29304(9)$ & $0.0117(3)$ \\
\hline $\mathrm{C} 17$ & $0.56598(8)$ & $0.33166(8)$ & $0.29579(9)$ & $0.0136(3)$ \\
\hline H17 & 0.6114 & 0.3250 & 0.2572 & $0.016^{*}$ \\
\hline $\mathrm{C} 22$ & $0.50242(8)$ & $0.20746(8)$ & $0.23027(9)$ & $0.0113(3)$ \\
\hline $\mathrm{C} 4$ & $-0.00106(8)$ & $0.28967(8)$ & $0.72282(9)$ & $0.0131(3)$ \\
\hline $\mathrm{C} 20$ & $0.43162(8)$ & $0.11255(8)$ & $0.13529(9)$ & $0.0141(3)$ \\
\hline $\mathrm{H} 20$ & 0.3814 & 0.0907 & 0.1139 & $0.017 *$ \\
\hline $\mathrm{C} 10$ & $-0.00090(8)$ & $0.22170(8)$ & $0.78738(9)$ & $0.0132(3)$ \\
\hline $\mathrm{C} 15$ & $0.43615(8)$ & $0.29027(8)$ & $0.35038(9)$ & $0.0146(3)$ \\
\hline H15 & 0.3911 & 0.2543 & 0.3504 & $0.018^{*}$ \\
\hline $\mathrm{C} 18$ & $0.56320(8)$ & $0.39594(8)$ & $0.35523(9)$ & $0.0125(3)$ \\
\hline H18 & 0.6076 & 0.4327 & 0.3569 & $0.015^{*}$ \\
\hline $\mathrm{C} 9$ & $0.07202(8)$ & $0.18464(9)$ & $0.81404(10)$ & $0.0164(3)$ \\
\hline H9 & 0.1227 & 0.2038 & 0.7920 & $0.020 *$ \\
\hline $\mathrm{C} 3$ & $-0.06460(8)$ & $0.34570(8)$ & $0.71891(9)$ & $0.0144(3)$ \\
\hline H3 & -0.1089 & 0.3418 & 0.7595 & $0.017 *$ \\
\hline
\end{tabular}




\begin{tabular}{|c|c|c|c|c|}
\hline $\mathrm{C} 21$ & $0.42956(8)$ & $0.17432(9)$ & $0.19781(9)$ & $0.0145(3)$ \\
\hline $\mathrm{H} 21$ & 0.3787 & 0.1941 & 0.2186 & $0.017 *$ \\
\hline $\mathrm{C} 14$ & $0.43721(8)$ & $0.35642(8)$ & $0.40738(9)$ & $0.0147(3)$ \\
\hline H14 & 0.3919 & 0.3654 & 0.4455 & $0.018^{*}$ \\
\hline $\mathrm{C} 8$ & $0.06999(8)$ & $0.11997(9)$ & $0.87270(10)$ & $0.0155(3)$ \\
\hline H8 & 0.1200 & 0.0952 & 0.8900 & $0.019^{*}$ \\
\hline $\mathrm{C} 5$ & $0.06262(8)$ & $0.29879(9)$ & $0.66202(10)$ & $0.0162(3)$ \\
\hline H5 & 0.1066 & 0.2616 & 0.6620 & $0.019^{*}$ \\
\hline $\mathrm{C} 24$ & $0.57206(8)$ & $0.11186(9)$ & $0.13636(10)$ & $0.0156(3)$ \\
\hline $\mathrm{H} 24$ & 0.6220 & 0.0897 & 0.1158 & $0.019^{*}$ \\
\hline $\mathrm{C} 23$ & $0.57484(8)$ & $0.17385(9)$ & $0.19922(10)$ & $0.0160(3)$ \\
\hline $\mathrm{H} 23$ & 0.6259 & 0.1933 & 0.2210 & $0.019^{*}$ \\
\hline $\mathrm{C} 11$ & $-0.07321(8)$ & 0.19167 (9) & $0.82304(9)$ & $0.0147(3)$ \\
\hline H11 & -0.1240 & 0.2156 & 0.8072 & $0.018 *$ \\
\hline $\mathrm{C} 12$ & $-0.07035(8)$ & $0.12663(9)$ & $0.88184(9)$ & $0.0142(3)$ \\
\hline H12 & -0.1200 & 0.1068 & 0.9057 & $0.017 *$ \\
\hline $\mathrm{C} 2$ & $-0.06252(8)$ & $0.40681(8)$ & $0.65549(9)$ & $0.0130(3)$ \\
\hline $\mathrm{H} 2$ & -0.1065 & 0.4440 & 0.6527 & $0.016^{*}$ \\
\hline $\mathrm{C} 45$ & $0.42153(9)$ & $0.96535(9)$ & 0.41679 (11) & $0.0208(3)$ \\
\hline H45 & 0.3752 & 0.9994 & 0.4215 & $0.025 *$ \\
\hline C6 & $0.06131(8)$ & 0.36237 (9) & $0.60178(10)$ & $0.0152(3)$ \\
\hline H6 & 0.1058 & 0.3687 & 0.5618 & $0.018 *$ \\
\hline $\mathrm{C} 46$ & $0.42614(9)$ & $0.89392(9)$ & $0.46622(10)$ & $0.0173(3)$ \\
\hline C44 & $0.48547(10)$ & $0.98589(10)$ & $0.36069(11)$ & $0.0226(3)$ \\
\hline H44 & 0.4811 & 1.0346 & 0.3273 & $0.027 *$ \\
\hline $\mathrm{C} 47$ & $0.49637(9)$ & $0.84765(9)$ & $0.45646(10)$ & $0.0201(3)$ \\
\hline H47 & 0.5025 & 0.7986 & 0.4889 & $0.024 *$ \\
\hline C 38 & $0.28158(9)$ & $0.76657(9)$ & $0.60272(10)$ & $0.0208(3)$ \\
\hline H38 & 0.2714 & 0.7110 & 0.6125 & $0.025 *$ \\
\hline N25 & $-0.12407(9)$ & $0.91210(10)$ & 0.49597 (11) & $0.0340(4)$ \\
\hline C39 & $0.34424(9)$ & $0.78762(9)$ & $0.54526(10)$ & 0.0195 (3) \\
\hline H39 & 0.3764 & 0.7470 & 0.5179 & $0.023 *$ \\
\hline $\mathrm{C} 40$ & $0.36000(9)$ & $0.86869(9)$ & $0.52772(10)$ & $0.0180(3)$ \\
\hline C36 & $0.22422(10)$ & 0.77389 (11) & $0.37480(11)$ & $0.0253(3)$ \\
\hline H36 & 0.2459 & 0.7215 & 0.3846 & $0.030 *$ \\
\hline $\mathrm{C} 48$ & $0.55719(9)$ & $0.87357(10)$ & 0.39917 (11) & $0.0222(3)$ \\
\hline H48 & 0.6047 & 0.8413 & 0.3941 & $0.027 *$ \\
\hline C34 & $0.11765(9)$ & $0.86961(10)$ & $0.40037(10)$ & $0.0224(3)$ \\
\hline $\mathrm{C} 35$ & $0.15162(9)$ & $0.79402(10)$ & $0.41632(11)$ & $0.0231(3)$ \\
\hline H35 & 0.1254 & 0.7568 & 0.4551 & $0.028 *$ \\
\hline C33 & $0.16047(10)$ & $0.92295(10)$ & $0.34582(11)$ & $0.0266(3)$ \\
\hline H33 & 0.1399 & 0.9754 & 0.3338 & $0.032 *$ \\
\hline C28 & $0.03559(10)$ & $0.88875(10)$ & 0.43575 (11) & $0.0232(3)$ \\
\hline C41 & $0.31092(10)$ & $0.92455(10)$ & $0.57120(12)$ & $0.0254(3)$ \\
\hline H41 & 0.3184 & 0.9805 & 0.5613 & $0.030 *$ \\
\hline $\mathrm{C} 27$ & $0.01285(10)$ & $0.86650(11)$ & 0.52287 (12) & $0.0286(4)$ \\
\hline $\mathrm{H} 27$ & 0.0513 & 0.8423 & 0.5630 & $0.034^{*}$ \\
\hline C32 & $0.23375(10)$ & $0.89772(11)$ & $0.30952(12)$ & $0.0286(4)$ \\
\hline
\end{tabular}




$\begin{array}{lllll}\text { H32 } & 0.2634 & 0.9349 & 0.2738 & 0.034^{*} \\ \text { C42 } & 0.25108(10) & 0.89764(10) & 0.62910(13) & 0.0299(4) \\ \text { H42 } & 0.2195 & 0.9370 & 0.6595 & 0.036^{*} \\ \text { C26 } & -0.06647(11) & 0.88027(12) & 0.54980(13) & 0.0335(4) \\ \text { H26 } & -0.0808 & 0.8663 & 0.6098 & 0.040^{*} \\ \text { C29 } & -0.02335(11) & 0.92411(11) & 0.38086(12) & 0.0302(4) \\ \text { H29 } & -0.0100 & 0.9422 & 0.3219 & 0.036^{*} \\ \text { C30 } & -0.10216(12) & 0.93284(12) & 0.41284(14) & 0.0367(4) \\ \text { H30 } & -0.1425 & 0.9547 & 0.3734 & 0.044^{*} \\ \text { H53B } & 0.2849(14) & 0.0514(14) & 0.7835(17) & 0.034(6)^{*} \\ \text { H52B } & 0.2595(15) & 0.1754(16) & 0.7175(17) & 0.046(7)^{*} \\ \text { H53A } & 0.3396(18) & 0.0590(18) & 0.720(2) & 0.066(9)^{*} \\ \text { H52A } & 0.2588(15) & 0.2311(15) & 0.6395(18) & 0.046(7)^{*} \\ \text { H50A } & 0.2641(16) & 0.1230(17) & 0.360(2) & 0.060(8)^{*} \\ \text { H49B } & 0.2334(18) & 0.0814(19) & 0.201(2) & 0.069(9)^{*} \\ \text { H50B } & 0.2281(19) & 0.0997(19) & 0.457(2) & 0.072(9)^{*} \\ \text { H49A } & 0.234(2) & 0.174(2) & 0.241(2) & 0.100(12)^{*} \\ \text { H51B } & 0.2650(13) & 0.2899(15) & 0.5027(14) & 0.034(6)^{*} \\ \text { H51A } & 0.281(2) & 0.207(2) & 0.480(2) & 0.093(1)^{*}\end{array}$

Atomic displacement parameters $\left(\AA^{2}\right)$

\begin{tabular}{lllllll}
\hline & $U^{11}$ & $U^{22}$ & $U^{33}$ & $U^{12}$ & $U^{13}$ & $U^{23}$ \\
\hline Cu1 & $0.01176(16)$ & $0.00446(15)$ & $0.00599(15)$ & $-0.00002(9)$ & $0.00097(11)$ & $0.00010(8)$ \\
Cu2 & $0.01341(16)$ & $0.00456(15)$ & $0.00628(15)$ & $0.00000(9)$ & $0.00100(11)$ & $-0.00001(8)$ \\
Si1 & $0.0105(2)$ & $0.0103(2)$ & $0.0118(2)$ & $0.00012(11)$ & $0.00098(16)$ & $-0.00039(12)$ \\
F1 & $0.0130(4)$ & $0.0140(5)$ & $0.0157(4)$ & $-0.0002(3)$ & $0.0016(3)$ & $-0.0021(3)$ \\
F2 & $0.0138(4)$ & $0.0154(5)$ & $0.0162(5)$ & $0.0010(3)$ & $0.0015(3)$ & $0.0020(3)$ \\
F5 & $0.0194(5)$ & $0.0653(8)$ & $0.0135(5)$ & $-0.0001(4)$ & $0.0007(4)$ & $0.0059(4)$ \\
F6 & $0.0196(5)$ & $0.0126(5)$ & $0.0616(7)$ & $-0.0008(3)$ & $0.0034(5)$ & $-0.0057(4)$ \\
F3 & $0.0187(5)$ & $0.0589(7)$ & $0.0139(5)$ & $0.0055(4)$ & $0.0012(4)$ & $-0.0002(4)$ \\
F4 & $0.0198(5)$ & $0.0123(5)$ & $0.0746(8)$ & $0.0007(3)$ & $0.0011(5)$ & $-0.0033(4)$ \\
N1 & $0.0130(5)$ & $0.0087(5)$ & $0.0096(5)$ & $-0.0006(4)$ & $0.0001(4)$ & $0.0019(4)$ \\
O52 & $0.0243(6)$ & $0.0258(6)$ & $0.0412(8)$ & $0.0045(5)$ & $0.0085(5)$ & $0.0034(5)$ \\
N7 & $0.0137(5)$ & $0.0086(5)$ & $0.0088(5)$ & $-0.0003(4)$ & $0.0007(4)$ & $0.0019(4)$ \\
N19 & $0.0145(5)$ & $0.0079(5)$ & $0.0085(5)$ & $0.0004(4)$ & $0.0004(4)$ & $-0.0010(4)$ \\
N13 & $0.0139(5)$ & $0.0072(5)$ & $0.0094(5)$ & $0.0003(4)$ & $0.0005(4)$ & $-0.0011(4)$ \\
O53 & $0.0346(7)$ & $0.0311(7)$ & $0.0302(7)$ & $0.0059(5)$ & $0.0124(5)$ & $0.0096(5)$ \\
O50 & $0.0271(6)$ & $0.0342(7)$ & $0.0366(7)$ & $0.0047(5)$ & $0.0013(5)$ & $-0.0021(5)$ \\
O51 & $0.0474(8)$ & $0.0258(7)$ & $0.0389(7)$ & $0.0018(6)$ & $-0.0021(6)$ & $0.0054(6)$ \\
N43 & $0.0228(6)$ & $0.0233(7)$ & $0.0212(6)$ & $-0.0028(5)$ & $0.0009(5)$ & $-0.0016(5)$ \\
O49 & $0.0472(8)$ & $0.0342(7)$ & $0.0310(7)$ & $0.0016(6)$ & $0.0001(6)$ & $0.0007(6)$ \\
N37 & $0.0198(6)$ & $0.0231(7)$ & $0.0305(7)$ & $-0.0016(5)$ & $0.0044(5)$ & $-0.0003(6)$ \\
N31 & $0.0206(6)$ & $0.0401(8)$ & $0.0238(7)$ & $-0.0031(6)$ & $0.0021(5)$ & $-0.0034(6)$ \\
C16 & $0.0136(6)$ & $0.0099(6)$ & $0.0118(6)$ & $0.0007(5)$ & $-0.0003(5)$ & $-0.0022(5)$ \\
C17 & $0.0134(6)$ & $0.0136(6)$ & $0.0138(6)$ & $-0.0008(5)$ & $0.0031(5)$ & $-0.0031(5)$ \\
C22 & $0.0142(6)$ & $0.0089(6)$ & $0.0109(6)$ & $-0.0007(5)$ & $0.0007(5)$ & $-0.0015(5)$ \\
C4 & $0.0139(6)$ & $0.0118(6)$ & $0.0137(6)$ & $-0.0001(5)$ & $0.0003(5)$ & $0.0042(5)$ \\
& & & & & &
\end{tabular}




\begin{tabular}{|c|c|c|c|c|c|c|}
\hline $\mathrm{C} 20$ & $0.0133(6)$ & $0.0127(6)$ & $0.0162(7)$ & $-0.0014(5)$ & $0.0012(5)$ & $-0.0040(5)$ \\
\hline $\mathrm{C} 10$ & $0.0148(6)$ & $0.0116(6)$ & $0.0132(6)$ & $0.0004(5)$ & $0.0016(5)$ & $0.0047(5)$ \\
\hline $\mathrm{C} 15$ & $0.0143(6)$ & $0.0126(6)$ & $0.0171(7)$ & $-0.0030(5)$ & $0.0032(5)$ & $-0.0043(5)$ \\
\hline C18 & $0.0140(6)$ & $0.0113(6)$ & $0.0122(6)$ & $-0.0010(5)$ & $0.0006(5)$ & $-0.0018(5)$ \\
\hline C9 & $0.0121(6)$ & $0.0179(7)$ & $0.0193(7)$ & $-0.0003(5)$ & $0.0022(5)$ & $0.0080(6)$ \\
\hline $\mathrm{C} 3$ & $0.0141(6)$ & $0.0155(7)$ & $0.0136(6)$ & $0.0015(5)$ & $0.0032(5)$ & $0.0042(5)$ \\
\hline $\mathrm{C} 21$ & $0.0126(6)$ & $0.0145(7)$ & $0.0164(7)$ & $0.0000(5)$ & $0.0022(5)$ & $-0.0045(5)$ \\
\hline C14 & $0.0159(6)$ & $0.0126(6)$ & $0.0157(6)$ & $-0.0011(5)$ & $0.0037(5)$ & $-0.0035(5)$ \\
\hline $\mathrm{C} 8$ & $0.0139(6)$ & $0.0148(7)$ & $0.0179(7)$ & $0.0016(5)$ & $0.0015(5)$ & $0.0058(5)$ \\
\hline $\mathrm{C} 5$ & $0.0131(6)$ & $0.0151(7)$ & $0.0205(7)$ & 0.0028 & $0.0032(5)$ & $0.0071(5)$ \\
\hline $\mathrm{C} 24$ & $0.0136(6)$ & $0.0154(7)$ & $0.0177(7)$ & $0.0016(5)$ & $0.0009(5)$ & $-0.0061(5)$ \\
\hline $\mathrm{C} 23$ & $0.0125(6)$ & $0.0175(7)$ & 0.0179 (7) & $-0.0002(5)$ & $-0.0012(5)$ & $-0.0067(5)$ \\
\hline C11 & $0.0119(6)$ & $0.0153(7)$ & $0.0168(6)$ & $0.0016(5)$ & $0.0009(5)$ & $0.0057(5)$ \\
\hline $\mathrm{C} 12$ & $0.0132(6)$ & $0.0148(7)$ & $0.0147(6)$ & $-0.0005(5)$ & $0.0008(5)$ & $0.0044(5)$ \\
\hline $\mathrm{C} 2$ & $0.0145(6)$ & $0.0128(6)$ & $0.0118(6)$ & $0.0018(5)$ & $0.0005(5)$ & $0.0018(5)$ \\
\hline $\mathrm{C} 45$ & $0.0192(7)$ & $0.0177(7)$ & $0.0255(8)$ & $0.0006(6)$ & $-0.0016(6)$ & $-0.0001(6)$ \\
\hline C6 & $0.0139(6)$ & $0.0145(7)$ & $0.0174(7)$ & $0.0014(5)$ & $0.0032(5)$ & $0.0058(5)$ \\
\hline $\mathrm{C} 46$ & $0.0176(7)$ & $0.0174(7)$ & $0.0169(7)$ & $-0.0015(5)$ & $-0.0028(5)$ & $-0.0021(5)$ \\
\hline $\mathrm{C} 44$ & $0.0252(8)$ & $0.0187(7)$ & $0.0237(8)$ & -0.0015 (6) & $-0.0007(6)$ & $0.0022(6)$ \\
\hline $\mathrm{C} 47$ & $0.0205(7)$ & $0.0192(7)$ & $0.0205(7)$ & $0.0017(6)$ & $-0.0016(6)$ & $0.0003(6)$ \\
\hline C38 & $0.0222(7)$ & $0.0182(7)$ & $0.0219(7)$ & $-0.0019(6)$ & $-0.0013(6)$ & $0.0005(6)$ \\
\hline $\mathrm{N} 25$ & $0.0270(7)$ & $0.0306(8)$ & $0.0446(9)$ & $0.0051(6)$ & $0.0069(7)$ & $-0.0021(7)$ \\
\hline C39 & $0.0228(7)$ & $0.0171(7)$ & $0.0186(7)$ & $0.0014(6)$ & $-0.0001(6)$ & $-0.0008(6)$ \\
\hline $\mathrm{C} 40$ & $0.0158(6)$ & $0.0190(7)$ & $0.0193(7)$ & $-0.0011(5)$ & $-0.0024(5)$ & $0.0000(6)$ \\
\hline C36 & 0.0198 (7) & $0.0319(9)$ & $0.0241(8)$ & 0.0008 (6) & $-0.0012(6)$ & $-0.0023(7)$ \\
\hline $\mathrm{C} 48$ & $0.0194(7)$ & $0.0242(8)$ & $0.0230(8)$ & $0.0018(6)$ & 0.0002 (6) & $-0.0020(6)$ \\
\hline C34 & $0.0214(7)$ & $0.0263(8)$ & $0.0194(7)$ & $-0.0025(6)$ & 0.0009 (6) & $-0.0037(6)$ \\
\hline C35 & $0.0203(7)$ & $0.0283(8)$ & $0.0205(7)$ & $-0.0026(6)$ & $-0.0004(6)$ & $0.0006(6)$ \\
\hline C33 & $0.0294(8)$ & $0.0249(8)$ & $0.0255(8)$ & -0.0039 & $0.0021(6)$ & $-0.0007(6)$ \\
\hline $\mathrm{C} 28$ & $0.0239(8)$ & $0.0210(8)$ & $0.0247(8)$ & -0.0023 & $0.0024(6)$ & $-0.0037(6)$ \\
\hline C41 & $0.0216(7)$ & $0.0160(7)$ & $0.0387(9)$ & $-0.0005(6)$ & $0.0060(7)$ & -0.0003 (6) \\
\hline $\mathrm{C} 27$ & $0.0259(8)$ & $0.0352(9)$ & $0.0246(8)$ & $0.0020(7)$ & $0.0019(6)$ & $0.0002(7)$ \\
\hline C32 & $0.0260(8)$ & $0.0347(9)$ & $0.0253(8)$ & $-0.0092(7)$ & $0.0043(6)$ & $-0.0018(7)$ \\
\hline $\mathrm{C} 42$ & $0.0239(8)$ & $0.0204(8)$ & $0.0455(10)$ & $0.0011(6)$ & $0.0122(7)$ & $-0.0028(7)$ \\
\hline $\mathrm{C} 26$ & $0.0317(9)$ & $0.0346(10)$ & $0.0343(10)$ & $0.0012(7)$ & $0.0113(7)$ & $0.0000(8)$ \\
\hline $\mathrm{C} 29$ & $0.0334(9)$ & $0.0264(9)$ & $0.0308(9)$ & $0.0047(7)$ & $0.0041(7)$ & $0.0029(7)$ \\
\hline $\mathrm{C} 30$ & $0.0318(9)$ & $0.0341(10)$ & $0.0442(11)$ & $0.0112(8)$ & $0.0010(8)$ & $0.0038(8)$ \\
\hline
\end{tabular}

Geometric parameters $\left(\AA,{ }^{\circ}\right)$

\begin{tabular}{llll}
\hline $\mathrm{Cu} 1-\mathrm{N} 1^{\mathrm{i}}$ & $2.0156(11)$ & $\mathrm{C} 10-\mathrm{C} 9$ & $1.3971(19)$ \\
$\mathrm{Cu} 1-\mathrm{N} 1$ & $2.0156(11)$ & $\mathrm{C} 15-\mathrm{C} 14$ & $1.3852(19)$ \\
$\mathrm{Cu} 1-\mathrm{N} 7^{\mathrm{ii}}$ & $2.0467(11)$ & $\mathrm{C} 15-\mathrm{H} 15$ & 0.9500 \\
$\mathrm{Cu} 1-\mathrm{N} 7^{\mathrm{iii}}$ & $2.0467(11)$ & $\mathrm{C} 18-\mathrm{H} 18$ & 0.9500 \\
$\mathrm{Cu} 1-\mathrm{F} 1^{\mathrm{i}}$ & $2.3585(9)$ & $\mathrm{C} 9-\mathrm{C} 8$ & $1.3818(19)$ \\
$\mathrm{Cu} 1-\mathrm{F} 1$ & $2.3585(9)$ & $\mathrm{C} 9-\mathrm{H} 9$ & 0.9500 \\
$\mathrm{Cu} 2-\mathrm{N} 13^{\text {iv }}$ & $2.0170(11)$ & $\mathrm{C} 3-\mathrm{C} 2$ & $1.3832(19)$ \\
$\mathrm{Cu} 2-\mathrm{N} 13$ & $2.0170(11)$ & $\mathrm{C} 3-\mathrm{H} 3$ & 0.9500
\end{tabular}




$$
\begin{aligned}
& \text { Cu2-N19 } \\
& \text { Cu2-N19 } \\
& \text { Cu2-F2 } \\
& \text { Cu2-F2 } \\
& \text { Si1-F6 } \\
& \text { Si1-F5 } \\
& \text { Si1-F3 } \\
& \text { Si1-F4 } \\
& \text { Si1-F2 } \\
& \text { Si1-F1 } \\
& \text { N1-C6 } \\
& \text { N1-C2 } \\
& \text { O52-H52B } \\
& \text { O52-H52A } \\
& \text { N7-C12 } \\
& \text { N7-C8 } \\
& \text { N7-Cu1 }{ }^{\text {vii }} \\
& \text { N19-C24 } \\
& \text { N19-C20 } \\
& \text { N19-Cu2 }{ }^{\text {viii }} \\
& \text { N13-C18 } \\
& \text { N13-C14 } \\
& \text { O53-H53B } \\
& \text { O53-H53A } \\
& \text { O50-H50A } \\
& \text { O50-H50B } \\
& \text { O51-H51B } \\
& \text { O51-H51A } \\
& \text { N43-C48 } \\
& \text { N43-C44 } \\
& \text { O49-H49B } \\
& \text { O49-H49A } \\
& \text { N37-C42 } \\
& \text { N37-C38 } \\
& \text { N31-C32 } \\
& \text { N31-C36 } \\
& \text { C16-C17 } \\
& \text { C16-C15 } \\
& \text { C16-C22 } \\
& \text { C17-C18 } \\
& \text { C17-H17 } \\
& \text { C22-C23 } \\
& \text { C22-C21 } \\
& \text { C4-C5 } \\
& \text { C4-C3 } \\
& \text { C20-C10 } 21 \\
&
\end{aligned}
$$

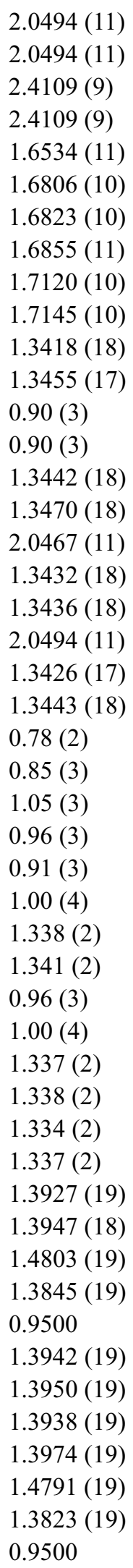

\begin{tabular}{|c|c|}
\hline $\mathrm{C} 21-\mathrm{H} 21$ & 0.9500 \\
\hline $\mathrm{C} 14-\mathrm{H} 14$ & 0.9500 \\
\hline $\mathrm{C} 8-\mathrm{H} 8$ & 0.9500 \\
\hline $\mathrm{C} 5-\mathrm{C} 6$ & $1.3821(19)$ \\
\hline $\mathrm{C} 5-\mathrm{H} 5$ & 0.9500 \\
\hline $\mathrm{C} 24-\mathrm{C} 23$ & $1.3882(19)$ \\
\hline $\mathrm{C} 24-\mathrm{H} 24$ & 0.9500 \\
\hline $\mathrm{C} 23-\mathrm{H} 23$ & 0.9500 \\
\hline $\mathrm{C} 11-\mathrm{C} 12$ & $1.3877(19)$ \\
\hline C11-H11 & 0.9500 \\
\hline $\mathrm{C} 12-\mathrm{H} 12$ & 0.9500 \\
\hline $\mathrm{C} 2-\mathrm{H} 2$ & 0.9500 \\
\hline $\mathrm{C} 45-\mathrm{C} 44$ & $1.387(2)$ \\
\hline $\mathrm{C} 45-\mathrm{C} 46$ & $1.396(2)$ \\
\hline $\mathrm{C} 45-\mathrm{H} 45$ & 0.9500 \\
\hline C6-H6 & 0.9500 \\
\hline $\mathrm{C} 46-\mathrm{C} 47$ & $1.393(2)$ \\
\hline $\mathrm{C} 46-\mathrm{C} 40$ & $1.484(2)$ \\
\hline $\mathrm{C} 44-\mathrm{H} 44$ & 0.9500 \\
\hline $\mathrm{C} 47-\mathrm{C} 48$ & $1.384(2)$ \\
\hline $\mathrm{C} 47-\mathrm{H} 47$ & 0.9500 \\
\hline C38-C39 & $1.385(2)$ \\
\hline C38-H38 & 0.9500 \\
\hline $\mathrm{N} 25-\mathrm{C} 30$ & $1.331(3)$ \\
\hline $\mathrm{N} 25-\mathrm{C} 26$ & $1.337(3)$ \\
\hline $\mathrm{C} 39-\mathrm{C} 40$ & $1.396(2)$ \\
\hline C39-H39 & 0.9500 \\
\hline $\mathrm{C} 40-\mathrm{C} 41$ & $1.391(2)$ \\
\hline $\mathrm{C} 36-\mathrm{C} 35$ & $1.386(2)$ \\
\hline C36-H36 & 0.9500 \\
\hline $\mathrm{C} 48-\mathrm{H} 48$ & 0.9500 \\
\hline C34-C35 & $1.393(2)$ \\
\hline C34-C33 & $1.394(2)$ \\
\hline $\mathrm{C} 34-\mathrm{C} 28$ & $1.483(2)$ \\
\hline C35-H35 & 0.9500 \\
\hline C33-C32 & $1.386(2)$ \\
\hline C33-H33 & 0.9500 \\
\hline $\mathrm{C} 28-\mathrm{C} 29$ & $1.386(2)$ \\
\hline $\mathrm{C} 28-\mathrm{C} 27$ & $1.396(2)$ \\
\hline $\mathrm{C} 41-\mathrm{C} 42$ & $1.384(2)$ \\
\hline $\mathrm{C} 41-\mathrm{H} 41$ & 0.9500 \\
\hline $\mathrm{C} 27-\mathrm{C} 26$ & $1.383(2)$ \\
\hline $\mathrm{C} 27-\mathrm{H} 27$ & 0.9500 \\
\hline C32-H32 & 0.9500 \\
\hline $\mathrm{C} 42-\mathrm{H} 42$ & 0.9500 \\
\hline $\mathrm{C} 26-\mathrm{H} 26$ & 0.9500 \\
\hline $\mathrm{C} 29-\mathrm{C} 30$ & $1.388(3)$ \\
\hline $\mathrm{C} 29-\mathrm{H} 29$ & 0.9500 \\
\hline
\end{tabular}


$\mathrm{C} 10-\mathrm{C} 11$

$\mathrm{N} 1-\mathrm{Cu} 1-\mathrm{N} 1$

$\mathrm{N} 1^{\mathrm{i}}-\mathrm{Cu} 1-\mathrm{N}^{\mathrm{ii}}$

$\mathrm{N} 1-\mathrm{Cu} 1-\mathrm{N} 7^{\mathrm{ii}}$

$\mathrm{N} 1^{\mathrm{i}}-\mathrm{Cu} 1-\mathrm{N} 7^{\mathrm{iii}}$

$\mathrm{N} 1-\mathrm{Cu} 1-\mathrm{N} 7^{\text {iii }}$

$\mathrm{N} 7^{\mathrm{ii}}-\mathrm{Cu} 1-\mathrm{N} 7^{\mathrm{iii}}$

$\mathrm{N} 1^{\mathrm{i}}-\mathrm{Cu} 1-\mathrm{F}^{\mathrm{i}}$

$\mathrm{N} 1-\mathrm{Cu} 1-\mathrm{F}^{\mathrm{i}}$

$\mathrm{N} 7^{\mathrm{ii}}-\mathrm{Cu} 1-\mathrm{F} 1^{\mathrm{i}}$

$\mathrm{N} 7^{\mathrm{iii}}-\mathrm{Cu} 1-\mathrm{F}^{\mathrm{i}}$

$\mathrm{N} 1-\mathrm{Cu} 1-\mathrm{F} 1$

$\mathrm{N} 1-\mathrm{Cu} 1-\mathrm{F} 1$

$\mathrm{N} 7 \mathrm{ii}-\mathrm{Cu} 1-\mathrm{F} 1$

$\mathrm{N} 7$ iii-Cu1-F1

$\mathrm{F} 1$ i- $\mathrm{Cu} 1-\mathrm{F} 1$

$\mathrm{N} 13^{\mathrm{iv}}-\mathrm{Cu} 2-\mathrm{N} 13$

$\mathrm{N} 13^{\mathrm{iv}}-\mathrm{Cu} 2-\mathrm{N} 19^{\mathrm{v}}$

$\mathrm{N} 13-\mathrm{Cu} 2-\mathrm{N} 19^{\mathrm{V}}$

$\mathrm{N} 13^{\text {iv }}-\mathrm{Cu} 2-\mathrm{N} 19^{\text {vi }}$

$\mathrm{N} 13-\mathrm{Cu} 2-\mathrm{N} 19^{\text {vi }}$

$\mathrm{N} 19^{v}-\mathrm{Cu} 2-\mathrm{N} 19^{\mathrm{vi}}$

$\mathrm{N} 13^{\mathrm{iv}}-\mathrm{Cu} 2-\mathrm{F} 2$

$\mathrm{N} 13-\mathrm{Cu} 2-\mathrm{F} 2$

$\mathrm{N} 19^{v}-\mathrm{Cu} 2-\mathrm{F} 2$

$\mathrm{N} 19^{\mathrm{vi}}-\mathrm{Cu} 2-\mathrm{F} 2$

$\mathrm{N} 13^{\mathrm{iv}}-\mathrm{Cu} 2-\mathrm{F}^{2 \mathrm{iv}}$

$\mathrm{N} 13-\mathrm{Cu} 2-\mathrm{F}^{\text {iv }}$

$\mathrm{N} 19^{v}-\mathrm{Cu} 2-\mathrm{F}^{\text {iv }}$

$\mathrm{N} 19^{\mathrm{vi}}-\mathrm{Cu} 2-\mathrm{F}^{\mathrm{iv}}$

$\mathrm{F} 2-\mathrm{Cu} 2-\mathrm{F}^{\mathrm{iv}}$

F6-Si1-F5

F6-Si1-F3

F5-Si1-F3

F6-Si1-F4

F5-Si1-F4

F3-Si1-F4

F6-Si1-F2

F5-Si1-F2

F3-Si1-F2

F4-Si1-F2

F6-Si1-F1

F5-Si1-F1

F3-Si1-F1

F4-Sil-F1

F2-Si1-F1

$\mathrm{Si} 1-\mathrm{F} 1-\mathrm{Cu} 1$

\section{$1.3948(19)$}

$180.00(6)$

$91.58(5)$

88.42 (5)

$88.42(5)$

$91.58(5)$

180.0

90.65 (4)

89.35 (4)

89.60 (4)

$90.40(4)$

89.35 (4)

90.65 (4)

90.40 (4)

89.60 (4)

180.0

180.0

$90.73(5)$

89.27 (5)

$89.27(5)$

$90.73(5)$

180.0

90.59 (4)

89.41 (4)

90.67 (4)

89.33 (4)

89.41 (4)

90.59 (4)

89.33 (4)

90.67 (4)

180.0

$90.80(6)$

$89.64(6)$

$179.55(6)$

178.87 (6)

$90.12(6)$

89.44 (6)

91.01 (4)

89.67 (5)

$90.41(5)$

89.64 (4)

90.89 (4)

$90.35(5)$

$89.56(5)$

88.45 (4)

178.09 (4)

$178.36(5)$
$\mathrm{C} 30-\mathrm{H} 30$

0.9500

119.53 (12)

120.2

120.2

119.74 (12)

120.1

120.1

122.35 (12)

118.8

118.8

122.68 (13)

118.7

118.7

119.54 (13)

120.2

120.2

$122.55(12)$

118.7

118.7

119.76 (12)

120.1

120.1

119.54 (12)

120.2

120.2

122.54 (12)

118.7

118.7

122.29 (12)

118.9

118.9

119.09 (14)

120.5

120.5

122.51 (12)

118.7

118.7

117.06 (14)

121.19 (14)

121.74 (13)

124.17 (15)

117.9

117.9

119.55 (14)

120.2

120.2

123.69 (14) 


\begin{tabular}{|c|c|c|c|}
\hline $\mathrm{Si} 1-\mathrm{F} 2-\mathrm{Cu} 2$ & $176.83(5)$ & $\mathrm{N} 37-\mathrm{C} 38-\mathrm{H} 38$ & 118.2 \\
\hline $\mathrm{C} 6-\mathrm{N} 1-\mathrm{C} 2$ & $118.46(12)$ & $\mathrm{C} 39-\mathrm{C} 38-\mathrm{H} 38$ & 118.2 \\
\hline $\mathrm{C} 6-\mathrm{N} 1-\mathrm{Cu} 1$ & $118.82(9)$ & $\mathrm{C} 30-\mathrm{N} 25-\mathrm{C} 26$ & $117.18(15)$ \\
\hline $\mathrm{C} 2-\mathrm{N} 1-\mathrm{Cu} 1$ & $122.58(9)$ & $\mathrm{C} 38-\mathrm{C} 39-\mathrm{C} 40$ & $119.83(14)$ \\
\hline $\mathrm{H} 52 \mathrm{~B}-\mathrm{O} 52-\mathrm{H} 52 \mathrm{~A}$ & $111(2)$ & $\mathrm{C} 38-\mathrm{C} 39-\mathrm{H} 39$ & 120.1 \\
\hline $\mathrm{C} 12-\mathrm{N} 7-\mathrm{C} 8$ & $118.09(12)$ & $\mathrm{C} 40-\mathrm{C} 39-\mathrm{H} 39$ & 120.1 \\
\hline $\mathrm{C} 12-\mathrm{N} 7-\mathrm{Cu} 1^{\mathrm{vii}}$ & $120.22(9)$ & $\mathrm{C} 41-\mathrm{C} 40-\mathrm{C} 39$ & $116.65(14)$ \\
\hline $\mathrm{C} 8-\mathrm{N} 7-\mathrm{Cu} 1^{\mathrm{vii}}$ & $121.50(9)$ & $\mathrm{C} 41-\mathrm{C} 40-\mathrm{C} 46$ & $121.72(14)$ \\
\hline $\mathrm{C} 24-\mathrm{N} 19-\mathrm{C} 20$ & $117.86(12)$ & $\mathrm{C} 39-\mathrm{C} 40-\mathrm{C} 46$ & $121.64(13)$ \\
\hline $\mathrm{C} 24-\mathrm{N} 19-\mathrm{Cu} 2^{\mathrm{viii}}$ & $121.30(9)$ & $\mathrm{N} 31-\mathrm{C} 36-\mathrm{C} 35$ & $123.33(16)$ \\
\hline $\mathrm{C} 20-\mathrm{N} 19-\mathrm{Cu} 2^{\text {viii }}$ & $120.62(9)$ & $\mathrm{N} 31-\mathrm{C} 36-\mathrm{H} 36$ & 118.3 \\
\hline $\mathrm{C} 18-\mathrm{N} 13-\mathrm{C} 14$ & $118.38(12)$ & $\mathrm{C} 35-\mathrm{C} 36-\mathrm{H} 36$ & 118.3 \\
\hline $\mathrm{C} 18-\mathrm{N} 13-\mathrm{Cu} 2$ & $121.49(9)$ & $\mathrm{N} 43-\mathrm{C} 48-\mathrm{C} 47$ & $123.95(14)$ \\
\hline $\mathrm{C} 14-\mathrm{N} 13-\mathrm{Cu} 2$ & $120.10(9)$ & $\mathrm{N} 43-\mathrm{C} 48-\mathrm{H} 48$ & 118.0 \\
\hline $\mathrm{H} 53 \mathrm{~B}-\mathrm{O} 53-\mathrm{H} 53 \mathrm{~A}$ & $108(3)$ & $\mathrm{C} 47-\mathrm{C} 48-\mathrm{H} 48$ & 118.0 \\
\hline $\mathrm{H} 50 \mathrm{~A}-\mathrm{O} 50-\mathrm{H} 50 \mathrm{~B}$ & $105(2)$ & $\mathrm{C} 35-\mathrm{C} 34-\mathrm{C} 33$ & $117.94(15)$ \\
\hline $\mathrm{H} 51 \mathrm{~B}-\mathrm{O} 51-\mathrm{H} 51 \mathrm{~A}$ & $98(2)$ & $\mathrm{C} 35-\mathrm{C} 34-\mathrm{C} 28$ & $119.72(14)$ \\
\hline $\mathrm{C} 48-\mathrm{N} 43-\mathrm{C} 44$ & $116.18(14)$ & $\mathrm{C} 33-\mathrm{C} 34-\mathrm{C} 28$ & $122.19(15)$ \\
\hline $\mathrm{H} 49 \mathrm{~B}-\mathrm{O} 49-\mathrm{H} 49 \mathrm{~A}$ & $114(3)$ & $\mathrm{C} 36-\mathrm{C} 35-\mathrm{C} 34$ & $119.07(15)$ \\
\hline $\mathrm{C} 42-\mathrm{N} 37-\mathrm{C} 38$ & $115.99(14)$ & $\mathrm{C} 36-\mathrm{C} 35-\mathrm{H} 35$ & 120.5 \\
\hline $\mathrm{C} 32-\mathrm{N} 31-\mathrm{C} 36$ & 117.09 (14) & $\mathrm{C} 34-\mathrm{C} 35-\mathrm{H} 35$ & 120.5 \\
\hline $\mathrm{C} 17-\mathrm{C} 16-\mathrm{C} 15$ & $117.72(12)$ & $\mathrm{C} 32-\mathrm{C} 33-\mathrm{C} 34$ & $118.45(16)$ \\
\hline $\mathrm{C} 17-\mathrm{C} 16-\mathrm{C} 22$ & $121.09(12)$ & $\mathrm{C} 32-\mathrm{C} 33-\mathrm{H} 33$ & 120.8 \\
\hline $\mathrm{C} 15-\mathrm{C} 16-\mathrm{C} 22$ & $121.19(12)$ & C34-C33-H33 & 120.8 \\
\hline $\mathrm{C} 18-\mathrm{C} 17-\mathrm{C} 16$ & $119.51(12)$ & $\mathrm{C} 29-\mathrm{C} 28-\mathrm{C} 27$ & $117.51(15)$ \\
\hline $\mathrm{C} 18-\mathrm{C} 17-\mathrm{H} 17$ & 120.2 & $\mathrm{C} 29-\mathrm{C} 28-\mathrm{C} 34$ & $120.85(15)$ \\
\hline $\mathrm{C} 16-\mathrm{C} 17-\mathrm{H} 17$ & 120.2 & $\mathrm{C} 27-\mathrm{C} 28-\mathrm{C} 34$ & $121.46(15)$ \\
\hline $\mathrm{C} 23-\mathrm{C} 22-\mathrm{C} 21$ & 117.19 (12) & $\mathrm{C} 42-\mathrm{C} 41-\mathrm{C} 40$ & $119.25(15)$ \\
\hline $\mathrm{C} 23-\mathrm{C} 22-\mathrm{C} 16$ & $122.08(12)$ & $\mathrm{C} 42-\mathrm{C} 41-\mathrm{H} 41$ & 120.4 \\
\hline $\mathrm{C} 21-\mathrm{C} 22-\mathrm{C} 16$ & $120.70(12)$ & $\mathrm{C} 40-\mathrm{C} 41-\mathrm{H} 41$ & 120.4 \\
\hline $\mathrm{C} 5-\mathrm{C} 4-\mathrm{C} 3$ & $117.65(12)$ & $\mathrm{C} 26-\mathrm{C} 27-\mathrm{C} 28$ & $118.95(17)$ \\
\hline $\mathrm{C} 5-\mathrm{C} 4-\mathrm{C} 10$ & $120.26(13)$ & $\mathrm{C} 26-\mathrm{C} 27-\mathrm{H} 27$ & 120.5 \\
\hline $\mathrm{C} 3-\mathrm{C} 4-\mathrm{C} 10$ & $122.08(12)$ & $\mathrm{C} 28-\mathrm{C} 27-\mathrm{H} 27$ & 120.5 \\
\hline $\mathrm{N} 19-\mathrm{C} 20-\mathrm{C} 21$ & $122.84(13)$ & $\mathrm{N} 31-\mathrm{C} 32-\mathrm{C} 33$ & $124.04(16)$ \\
\hline $\mathrm{N} 19-\mathrm{C} 20-\mathrm{H} 20$ & 118.6 & $\mathrm{~N} 31-\mathrm{C} 32-\mathrm{H} 32$ & 118.0 \\
\hline $\mathrm{C} 21-\mathrm{C} 20-\mathrm{H} 20$ & 118.6 & $\mathrm{C} 33-\mathrm{C} 32-\mathrm{H} 32$ & 118.0 \\
\hline $\mathrm{C} 11-\mathrm{C} 10-\mathrm{C} 9$ & $117.59(12)$ & $\mathrm{N} 37-\mathrm{C} 42-\mathrm{C} 41$ & $124.56(15)$ \\
\hline $\mathrm{C} 11-\mathrm{C} 10-\mathrm{C} 4$ & $121.48(13)$ & $\mathrm{N} 37-\mathrm{C} 42-\mathrm{H} 42$ & 117.7 \\
\hline $\mathrm{C} 9-\mathrm{C} 10-\mathrm{C} 4$ & $120.93(12)$ & $\mathrm{C} 41-\mathrm{C} 42-\mathrm{H} 42$ & 117.7 \\
\hline $\mathrm{C} 14-\mathrm{C} 15-\mathrm{C} 16$ & $119.54(13)$ & $\mathrm{N} 25-\mathrm{C} 26-\mathrm{C} 27$ & $123.59(17)$ \\
\hline $\mathrm{C} 14-\mathrm{C} 15-\mathrm{H} 15$ & 120.2 & $\mathrm{~N} 25-\mathrm{C} 26-\mathrm{H} 26$ & 118.2 \\
\hline $\mathrm{C} 16-\mathrm{C} 15-\mathrm{H} 15$ & 120.2 & $\mathrm{C} 27-\mathrm{C} 26-\mathrm{H} 26$ & 118.2 \\
\hline $\mathrm{N} 13-\mathrm{C} 18-\mathrm{C} 17$ & $122.50(12)$ & $\mathrm{C} 28-\mathrm{C} 29-\mathrm{C} 30$ & $119.30(17)$ \\
\hline $\mathrm{N} 13-\mathrm{C} 18-\mathrm{H} 18$ & 118.8 & $\mathrm{C} 28-\mathrm{C} 29-\mathrm{H} 29$ & 120.3 \\
\hline $\mathrm{C} 17-\mathrm{C} 18-\mathrm{H} 18$ & 118.8 & $\mathrm{C} 30-\mathrm{C} 29-\mathrm{H} 29$ & 120.3 \\
\hline $\mathrm{C} 8-\mathrm{C} 9-\mathrm{C} 10$ & $119.56(13)$ & $\mathrm{N} 25-\mathrm{C} 30-\mathrm{C} 29$ & $123.38(18)$ \\
\hline $\mathrm{C} 8-\mathrm{C} 9-\mathrm{H} 9$ & 120.2 & $\mathrm{~N} 25-\mathrm{C} 30-\mathrm{H} 30$ & 118.3 \\
\hline $\mathrm{C} 10-\mathrm{C} 9-\mathrm{H} 9$ & 120.2 & $\mathrm{C} 29-\mathrm{C} 30-\mathrm{H} 30$ & 118.3 \\
\hline
\end{tabular}




\begin{tabular}{|c|c|c|c|}
\hline $\mathrm{C} 15-\mathrm{C} 16-\mathrm{C} 17-\mathrm{C} 18$ & $0.9(2)$ & $\mathrm{C} 4-\mathrm{C} 3-\mathrm{C} 2-\mathrm{N} 1$ & $-1.1(2)$ \\
\hline $\mathrm{C} 22-\mathrm{C} 16-\mathrm{C} 17-\mathrm{C} 18$ & $179.87(12)$ & $\mathrm{C} 2-\mathrm{N} 1-\mathrm{C} 6-\mathrm{C} 5$ & $1.0(2)$ \\
\hline $\mathrm{C} 17-\mathrm{C} 16-\mathrm{C} 22-\mathrm{C} 23$ & $26.6(2)$ & $\mathrm{Cu} 1-\mathrm{N} 1-\mathrm{C} 6-\mathrm{C} 5$ & $-174.86(11)$ \\
\hline $\mathrm{C} 15-\mathrm{C} 16-\mathrm{C} 22-\mathrm{C} 23$ & $-154.49(14)$ & $\mathrm{C} 4-\mathrm{C} 5-\mathrm{C} 6-\mathrm{N} 1$ & $-1.6(2)$ \\
\hline $\mathrm{C} 17-\mathrm{C} 16-\mathrm{C} 22-\mathrm{C} 21$ & $-151.72(14)$ & $\mathrm{C} 44-\mathrm{C} 45-\mathrm{C} 46-\mathrm{C} 47$ & $0.7(2)$ \\
\hline $\mathrm{C} 15-\mathrm{C} 16-\mathrm{C} 22-\mathrm{C} 21$ & $27.22(19)$ & $\mathrm{C} 44-\mathrm{C} 45-\mathrm{C} 46-\mathrm{C} 40$ & $179.73(14)$ \\
\hline $\mathrm{C} 24-\mathrm{N} 19-\mathrm{C} 20-\mathrm{C} 21$ & $2.0(2)$ & $\mathrm{C} 48-\mathrm{N} 43-\mathrm{C} 44-\mathrm{C} 45$ & $-0.5(2)$ \\
\hline 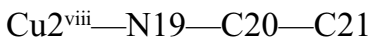 & $-172.69(11)$ & $\mathrm{C} 46-\mathrm{C} 45-\mathrm{C} 44-\mathrm{N} 43$ & $-0.3(2)$ \\
\hline $\mathrm{C} 5-\mathrm{C} 4-\mathrm{C} 10-\mathrm{C} 11$ & $-154.60(14)$ & $\mathrm{C} 45-\mathrm{C} 46-\mathrm{C} 47-\mathrm{C} 48$ & $-0.3(2)$ \\
\hline $\mathrm{C} 3-\mathrm{C} 4-\mathrm{C} 10-\mathrm{C} 11$ & $24.2(2)$ & $\mathrm{C} 40-\mathrm{C} 46-\mathrm{C} 47-\mathrm{C} 48$ & $-179.31(14)$ \\
\hline $\mathrm{C} 5-\mathrm{C} 4-\mathrm{C} 10-\mathrm{C} 9$ & $24.7(2)$ & $\mathrm{C} 42-\mathrm{N} 37-\mathrm{C} 38-\mathrm{C} 39$ & $0.3(2)$ \\
\hline $\mathrm{C} 3-\mathrm{C} 4-\mathrm{C} 10-\mathrm{C} 9$ & $-156.55(14)$ & $\mathrm{N} 37-\mathrm{C} 38-\mathrm{C} 39-\mathrm{C} 40$ & $-1.4(2)$ \\
\hline $\mathrm{C} 17-\mathrm{C} 16-\mathrm{C} 15-\mathrm{C} 14$ & $-0.2(2)$ & $\mathrm{C} 38-\mathrm{C} 39-\mathrm{C} 40-\mathrm{C} 41$ & $0.7(2)$ \\
\hline $\mathrm{C} 22-\mathrm{C} 16-\mathrm{C} 15-\mathrm{C} 14$ & $-179.17(13)$ & $\mathrm{C} 38-\mathrm{C} 39-\mathrm{C} 40-\mathrm{C} 46$ & $-179.63(13)$ \\
\hline $\mathrm{C} 14-\mathrm{N} 13-\mathrm{C} 18-\mathrm{C} 17$ & $-0.5(2)$ & $\mathrm{C} 47-\mathrm{C} 46-\mathrm{C} 40-\mathrm{C} 41$ & $149.49(16)$ \\
\hline $\mathrm{Cu} 2-\mathrm{N} 13-\mathrm{C} 18-\mathrm{C} 17$ & $177.47(10)$ & $\mathrm{C} 45-\mathrm{C} 46-\mathrm{C} 40-\mathrm{C} 41$ & $-29.5(2)$ \\
\hline $\mathrm{C} 16-\mathrm{C} 17-\mathrm{C} 18-\mathrm{N} 13$ & $-0.6(2)$ & $\mathrm{C} 47-\mathrm{C} 46-\mathrm{C} 40-\mathrm{C} 39$ & $-30.1(2)$ \\
\hline $\mathrm{C} 11-\mathrm{C} 10-\mathrm{C} 9-\mathrm{C} 8$ & $0.9(2)$ & $\mathrm{C} 45-\mathrm{C} 46-\mathrm{C} 40-\mathrm{C} 39$ & $150.90(15)$ \\
\hline $\mathrm{C} 4-\mathrm{C} 10-\mathrm{C} 9-\mathrm{C} 8$ & $-178.38(14)$ & $\mathrm{C} 32-\mathrm{N} 31-\mathrm{C} 36-\mathrm{C} 35$ & $0.5(2)$ \\
\hline $\mathrm{C} 5-\mathrm{C} 4-\mathrm{C} 3-\mathrm{C} 2$ & $0.5(2)$ & $\mathrm{C} 44-\mathrm{N} 43-\mathrm{C} 48-\mathrm{C} 47$ & $1.0(2)$ \\
\hline $\mathrm{C} 10-\mathrm{C} 4-\mathrm{C} 3-\mathrm{C} 2$ & $-178.27(13)$ & $\mathrm{C} 46-\mathrm{C} 47-\mathrm{C} 48-\mathrm{N} 43$ & $-0.6(2)$ \\
\hline $\mathrm{N} 19-\mathrm{C} 20-\mathrm{C} 21-\mathrm{C} 22$ & $0.0(2)$ & $\mathrm{N} 31-\mathrm{C} 36-\mathrm{C} 35-\mathrm{C} 34$ & $2.0(2)$ \\
\hline $\mathrm{C} 23-\mathrm{C} 22-\mathrm{C} 21-\mathrm{C} 20$ & $-2.0(2)$ & $\mathrm{C} 33-\mathrm{C} 34-\mathrm{C} 35-\mathrm{C} 36$ & $-2.6(2)$ \\
\hline $\mathrm{C} 16-\mathrm{C} 22-\mathrm{C} 21-\mathrm{C} 20$ & $176.33(13)$ & $\mathrm{C} 28-\mathrm{C} 34-\mathrm{C} 35-\mathrm{C} 36$ & $173.10(15)$ \\
\hline $\mathrm{C} 18-\mathrm{N} 13-\mathrm{C} 14-\mathrm{C} 15$ & $1.2(2)$ & $\mathrm{C} 35-\mathrm{C} 34-\mathrm{C} 33-\mathrm{C} 32$ & $0.8(2)$ \\
\hline $\mathrm{Cu} 2-\mathrm{N} 13-\mathrm{C} 14-\mathrm{C} 15$ & $-176.76(11)$ & $\mathrm{C} 28-\mathrm{C} 34-\mathrm{C} 33-\mathrm{C} 32$ & $-174.74(15)$ \\
\hline $\mathrm{C} 16-\mathrm{C} 15-\mathrm{C} 14-\mathrm{N} 13$ & $-0.9(2)$ & $\mathrm{C} 35-\mathrm{C} 34-\mathrm{C} 28-\mathrm{C} 29$ & $-132.33(17)$ \\
\hline $\mathrm{C} 12-\mathrm{N} 7-\mathrm{C} 8-\mathrm{C} 9$ & $-0.4(2)$ & $\mathrm{C} 33-\mathrm{C} 34-\mathrm{C} 28-\mathrm{C} 29$ & $43.2(2)$ \\
\hline $\mathrm{Cu} 1^{\mathrm{vii}}-\mathrm{N} 7-\mathrm{C} 8-\mathrm{C} 9$ & $-175.57(11)$ & $\mathrm{C} 35-\mathrm{C} 34-\mathrm{C} 28-\mathrm{C} 27$ & $42.8(2)$ \\
\hline $\mathrm{C} 10-\mathrm{C} 9-\mathrm{C} 8-\mathrm{N} 7$ & $-0.4(2)$ & $\mathrm{C} 33-\mathrm{C} 34-\mathrm{C} 28-\mathrm{C} 27$ & $-141.73(17)$ \\
\hline $\mathrm{C} 3-\mathrm{C} 4-\mathrm{C} 5-\mathrm{C} 6$ & $0.8(2)$ & $\mathrm{C} 39-\mathrm{C} 40-\mathrm{C} 41-\mathrm{C} 42$ & $0.8(2)$ \\
\hline $\mathrm{C} 10-\mathrm{C} 4-\mathrm{C} 5-\mathrm{C} 6$ & $179.62(13)$ & $\mathrm{C} 46-\mathrm{C} 40-\mathrm{C} 41-\mathrm{C} 42$ & $-178.85(15)$ \\
\hline $\mathrm{C} 20-\mathrm{N} 19-\mathrm{C} 24-\mathrm{C} 23$ & $-2.0(2)$ & $\mathrm{C} 29-\mathrm{C} 28-\mathrm{C} 27-\mathrm{C} 26$ & $0.3(3)$ \\
\hline 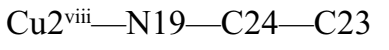 & $172.69(11)$ & $\mathrm{C} 34-\mathrm{C} 28-\mathrm{C} 27-\mathrm{C} 26$ & $-174.98(16)$ \\
\hline $\mathrm{N} 19-\mathrm{C} 24-\mathrm{C} 23-\mathrm{C} 22$ & $-0.1(2)$ & $\mathrm{C} 36-\mathrm{N} 31-\mathrm{C} 32-\mathrm{C} 33$ & $-2.4(3)$ \\
\hline $\mathrm{C} 21-\mathrm{C} 22-\mathrm{C} 23-\mathrm{C} 24$ & $2.1(2)$ & $\mathrm{C} 34-\mathrm{C} 33-\mathrm{C} 32-\mathrm{N} 31$ & $1.8(3)$ \\
\hline $\mathrm{C} 16-\mathrm{C} 22-\mathrm{C} 23-\mathrm{C} 24$ & $-176.27(13)$ & $\mathrm{C} 38-\mathrm{N} 37-\mathrm{C} 42-\mathrm{C} 41$ & $1.3(3)$ \\
\hline $\mathrm{C} 9-\mathrm{C} 10-\mathrm{C} 11-\mathrm{C} 12$ & $-0.8(2)$ & $\mathrm{C} 40-\mathrm{C} 41-\mathrm{C} 42-\mathrm{N} 37$ & $-1.9(3)$ \\
\hline $\mathrm{C} 4-\mathrm{C} 10-\mathrm{C} 11-\mathrm{C} 12$ & $178.56(13)$ & $\mathrm{C} 30-\mathrm{N} 25-\mathrm{C} 26-\mathrm{C} 27$ & $-1.5(3)$ \\
\hline $\mathrm{C} 8-\mathrm{N} 7-\mathrm{C} 12-\mathrm{C} 11$ & $0.6(2)$ & $\mathrm{C} 28-\mathrm{C} 27-\mathrm{C} 26-\mathrm{N} 25$ & $1.8(3)$ \\
\hline $\mathrm{Cu} 1$ vii_-N7-C12-C11 & $175.83(11)$ & $\mathrm{C} 27-\mathrm{C} 28-\mathrm{C} 29-\mathrm{C} 30$ & $-2.6(3)$ \\
\hline $\mathrm{C} 10-\mathrm{C} 11-\mathrm{C} 12-\mathrm{N} 7$ & $0.0(2)$ & $\mathrm{C} 34-\mathrm{C} 28-\mathrm{C} 29-\mathrm{C} 30$ & $172.72(17)$ \\
\hline $\mathrm{C} 6-\mathrm{N} 1-\mathrm{C} 2-\mathrm{C} 3$ & $0.4(2)$ & $\mathrm{C} 26-\mathrm{N} 25-\mathrm{C} 30-\mathrm{C} 29$ & $-1.0(3)$ \\
\hline $\mathrm{Cu} 1-\mathrm{N} 1-\mathrm{C} 2-\mathrm{C} 3$ & $176.11(10)$ & $\mathrm{C} 28-\mathrm{C} 29-\mathrm{C} 30-\mathrm{N} 25$ & $3.1(3)$ \\
\hline
\end{tabular}

Symmetry codes: (i) $-x,-y+1,-z+1$; (ii) $x,-y+1 / 2, z-1 / 2$; (iii) $-x, y+1 / 2,-z+3 / 2$; (iv) $-x+1,-y+1,-z+1$; (v) $x,-y+1 / 2, z+1 / 2$; (vi) $-x+1, y+1 / 2,-z+1 / 2$; (vii) $-x, y-1 / 2,-z+3 / 2$; (viii) $-x+1, y-1 / 2,-z+1 / 2$. 
Hydrogen-bond geometry $\left(\AA,{ }^{\circ}\right)$

\begin{tabular}{|c|c|c|c|c|}
\hline$D-\mathrm{H} \cdots A$ & $D-\mathrm{H}$ & $\mathrm{H} \cdots A$ & $D \cdots A$ & $D-\mathrm{H} \cdots A$ \\
\hline $\mathrm{C} 17-\mathrm{H} 17 \cdots \mathrm{N} 31^{\text {viii }}$ & 0.95 & 2.34 & $3.2849(19)$ & 170 \\
\hline $\mathrm{C} 20-\mathrm{H} 20 \cdots \mathrm{F} 2^{\mathrm{ii}}$ & 0.95 & 2.32 & $3.0341(16)$ & 131 \\
\hline $\mathrm{C} 20-\mathrm{H} 20 \cdots \mathrm{F}^{\mathrm{ii}}$ & 0.95 & 2.53 & $3.4305(17)$ & 159 \\
\hline $\mathrm{C} 18-\mathrm{H} 18 \cdots \mathrm{F} 2^{\mathrm{iv}}$ & 0.95 & 2.52 & $3.1085(16)$ & 120 \\
\hline $\mathrm{C} 9-\mathrm{H} 9 \cdots \mathrm{O} 52$ & 0.95 & 2.50 & $3.4223(18)$ & 163 \\
\hline $\mathrm{C} 3-\mathrm{H} 3 \cdots \mathrm{N} 37^{\mathrm{vii}}$ & 0.95 & 2.54 & $3.4803(19)$ & 173 \\
\hline $\mathrm{C} 21-\mathrm{H} 21 \cdots \mathrm{O} 52^{\mathrm{ii}}$ & 0.95 & 2.56 & $3.4294(18)$ & 152 \\
\hline $\mathrm{C} 14-\mathrm{H} 14 \cdots \mathrm{F} 2$ & 0.95 & 2.43 & $3.0497(16)$ & 123 \\
\hline $\mathrm{C} 14-\mathrm{H} 14 \cdots \mathrm{F} 4$ & 0.95 & 2.55 & $3.4519(17)$ & 158 \\
\hline $\mathrm{C} 8-\mathrm{H} 8 \cdots \mathrm{F} 1^{\mathrm{v}}$ & 0.95 & 2.33 & $3.0205(16)$ & 129 \\
\hline $\mathrm{C} 8-\mathrm{H} 8 \cdots \mathrm{F}^{\mathrm{v}}$ & 0.95 & 2.51 & $3.3524(18)$ & 148 \\
\hline $\mathrm{C} 5-\mathrm{H} 5 \cdots \mathrm{O} 52$ & 0.95 & 2.43 & $3.2916(18)$ & 151 \\
\hline $\mathrm{C} 24-\mathrm{H} 24 \cdots \mathrm{F} 2^{\mathrm{viii}}$ & 0.95 & 2.29 & $2.9997(16)$ & 131 \\
\hline $\mathrm{C} 24-\mathrm{H} 24 \cdots \mathrm{F}^{\mathrm{viii}}$ & 0.95 & 2.55 & $3.4609(17)$ & 161 \\
\hline $\mathrm{C} 11-\mathrm{H} 11 \cdots \mathrm{N} 37^{\mathrm{vii}}$ & 0.95 & 2.61 & $3.4358(19)$ & 145 \\
\hline $\mathrm{C} 12-\mathrm{H} 12 \cdots \mathrm{F} 1^{\mathrm{vii}}$ & 0.95 & 2.26 & $2.9662(16)$ & 131 \\
\hline $\mathrm{C} 12-\mathrm{H} 12 \cdots \mathrm{F}^{\mathrm{vii}}$ & 0.95 & 2.49 & $3.4040(16)$ & 161 \\
\hline $\mathrm{C} 2-\mathrm{H} 2 \cdots \mathrm{F} 1^{\mathrm{i}}$ & 0.95 & 2.52 & $3.0708(16)$ & 117 \\
\hline $\mathrm{C} 2-\mathrm{H} 2 \cdots \mathrm{F} 3^{\mathrm{i}}$ & 0.95 & 2.45 & $3.3364(17)$ & 156 \\
\hline $\mathrm{C} 45-\mathrm{H} 45 \cdots \mathrm{O} 50^{\text {ix }}$ & 0.95 & 2.44 & $3.376(2)$ & 167 \\
\hline $\mathrm{C} 6-\mathrm{H} 6 \cdots \mathrm{F} 1$ & 0.95 & 2.46 & $3.0691(16)$ & 122 \\
\hline $\mathrm{C} 6-\mathrm{H} 6 \cdots \mathrm{F} 4$ & 0.95 & 2.60 & $3.5313(17)$ & 166 \\
\hline $\mathrm{C} 38-\mathrm{H} 38 \cdots \mathrm{F} 6$ & 0.95 & 2.41 & $3.1240(19)$ & 132 \\
\hline $\mathrm{C} 48-\mathrm{H} 48 \cdots \mathrm{O} 51^{\mathrm{iv}}$ & 0.95 & 2.50 & $3.330(2)$ & 146 \\
\hline $\mathrm{O} 53-\mathrm{H} 53 B \cdots \mathrm{F} 3^{v}$ & $0.78(2)$ & $1.96(3)$ & $2.7228(17)$ & $166(2)$ \\
\hline $\mathrm{O} 52-\mathrm{H} 52 B \cdots \mathrm{O} 53$ & $0.90(3)$ & $1.83(3)$ & $2.7227(18)$ & $170(2)$ \\
\hline $\mathrm{O} 53-\mathrm{H} 53 A \cdots \mathrm{N} 43^{\mathrm{iv}}$ & $0.85(3)$ & $2.05(3)$ & $2.8666(19)$ & $161(3)$ \\
\hline $\mathrm{O} 52-\mathrm{H} 52 A \cdots \mathrm{O} 51$ & $0.90(3)$ & $1.84(3)$ & $2.726(2)$ & $172(2)$ \\
\hline $\mathrm{O} 50-\mathrm{H} 50 A \cdots \mathrm{O} 49$ & $1.05(3)$ & $1.72(3)$ & $2.741(2)$ & $163(2)$ \\
\hline $\mathrm{O} 49-\mathrm{H} 49 B \cdots \mathrm{F} 5^{\mathrm{ii}}$ & $0.96(3)$ & $1.82(3)$ & $2.7716(18)$ & $173(3)$ \\
\hline $\mathrm{O} 50-\mathrm{H} 50 B^{\cdots} \cdots \mathrm{N} 25^{\mathrm{i}}$ & $0.96(3)$ & $1.86(3)$ & $2.816(2)$ & $173(3)$ \\
\hline $\mathrm{O} 49-\mathrm{H} 49 A \cdots \mathrm{O} 52^{\mathrm{ii}}$ & $1.00(4)$ & $1.81(4)$ & $2.798(2)$ & $169(3)$ \\
\hline $\mathrm{O} 51-\mathrm{H} 51 B \cdots \mathrm{F} 4$ & $0.91(3)$ & $1.88(3)$ & $2.7029(18)$ & $149(2)$ \\
\hline $\mathrm{O} 51-\mathrm{H} 51 A^{\cdots} \mathrm{O} 50$ & $1.00(4)$ & $1.76(4)$ & $2.719(2)$ & $158(3)$ \\
\hline
\end{tabular}

Symmetry codes: (i) $-x,-y+1,-z+1$; (ii) $x,-y+1 / 2, z-1 / 2$; (iv) $-x+1,-y+1,-z+1$; (v) $x,-y+1 / 2, z+1 / 2$; (vii) $-x, y-1 / 2,-z+3 / 2$; (viii) $-x+1, y-1 / 2,-z+1 / 2$; (ix) $x, y+1, z$. 